BONN-TH-94-007

\title{
MIRROR SYMMETRY AND STRING VACUA FROM A SPECIAL CLASS OF FANO VARIETIES
}

\author{
Rolf SCHIMMrigk \\ Physikalisches Institut, Universität Bonn \\ Nussallee 12, 53115 Bonn, FRG
}

\begin{abstract}
Because of the existence of rigid Calabi-Yau manifolds, mirror symmetry cannot be understood as an operation on the space of manifolds with vanishing first Chern class. In this article I continue to investigate a particular type of Kähler manifolds with positive first Chern class which generalize Calabi-Yau manifolds in a natural way and which provide a framework for mirrors of rigid string vacua. This class is comprised of a special type of Fano manifolds which encode crucial information about ground states of the superstring. It is shown in particular that the massless spectra of $(2,2)$-supersymmetric vacua of central charge $\hat{c}=D_{\text {crit }}$ can be derived from special Fano varieties of complex dimension $\left(D_{\text {crit }}+2(Q-1)\right), Q>1$, and that in certain circumstances it is even possible to embed Calabi-Yau manifolds into such higher dimensional spaces. The constructions described here lead to new insight into the relation between exactly solvable models and their mean field theories on the one hand and their corresponding Calabi-Yau manifolds on the other. It is furthermore shown that Witten's formulation of the Landau-Ginzburg/Calabi-Yau relation can be applied to the present framework as well.
\end{abstract}

\footnotetext{
${ }^{\diamond}$ Email address: netah@avzw02.physik.uni-bonn.de
} 


\section{Contents}

1. Introduction

2. A Special Class of Fano Manifolds with Quantized Positive $1^{\text {st }}$ Chern Class

3. Special Fano Varieties and Critical String Vacua: the Physical Dimension

4. Generalization to Arbitrary Critical Dimension

5. Cohomology of Special Fano Spaces and Critical Spectra

6. When $h^{D_{\text {crit }}+Q-1, Q-1}>1$ : A Degeneration Phenomenon

7. Splitting and Contraction - Many Fano Fold Embeddings of Critical Vacua

8. Topological Relations between Fano Varieties

9. Landau-Ginzburg Theories, Special Fano Manifolds, and Critical Vacua

10. Mirrors of Rigid String Vacua

11. Toric Considerations

12. Phases of Special Fano Manifolds

13. Special Complete Intersection Fano Manifolds

14. Conclusion 


\section{Introduction}

The discovery of mirror symmetry [1] 2] [3] has made it possible to address a number of longstanding issues in string theory. Some of the most important ones are concerned with questions regarding the correct geometrical framework of string compactification, the occurrence of topology change and the appearance of a minimal distance in string theory, the action of modular groups on moduli space and, finally, the computation of exact Yukawa couplings not only at special points in the moduli space but as functions of the moduli. The latter application is of interest in particular for concrete application to physics as well as in mathematics: on the physical side it in fact pre-empties one of the major motivations for considering string vacua constructed from exactly solvable models whereas on the mathematical side it allows to obtain results that were thought completely out of reach prior to the advent of mirror symmetry.

It is not easy, surprisingly, to say, in full generality, what mirror symmetry is without reference to any particular framework for string compactification. As a first step one may consider the conventional framework, formulated in [4], in which left-right symmetric compactifications without torsion are described by internal spaces which are compact complex Kähler manifolds and admit a covariantly constant spinor, i.e. have vanishing first Chern class, so-called Calabi-Yau manifolds. Such spaces are particularly simple, a fact that is encoded concisely in the spectrum of the theory, parametrized, in part, by the cohomology of the manifolds. Because the internal space is complex, the real cohomology can be decomposed, via the Hodge decomposition, into complex cohomology groups. Thus the Betti numbers $b_{i}=\operatorname{dim} H^{i}(\mathcal{M}, \mathbb{R})$ can be expressed in terms of the Hodge numbers $h^{(p, q)}=\operatorname{dim} H^{p, q}(\mathcal{M}, \mathbb{C}): b_{i}=\sum_{p+q=i} h^{(p, q)}$. Since the manifold is Kähler, the Hodge numbers are symmetric, $h^{(p, q)}=h^{(q, p)}$, and because the first Chern class vanishes, it follows that $h^{(i, 0)}=0=h^{(0, i)}, i=1,2$ and $h^{(3,0)}=1=h^{(0,3)}$. Hence the cohomology of the internal space, summarized in the Hodge diamond

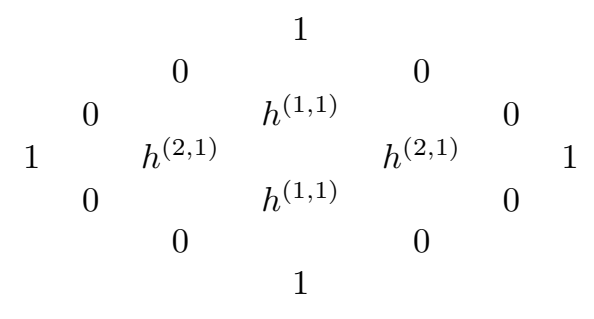

contains only two independent elements $h^{(1,1)}=h^{(2,2)}$ and $h^{(2,1)}=h^{(1,2)}$ which parametrize the number of antigenerations and generations, respectively, that we observe in low energy physics. 
It has become clear through the work of Gepner [5] [6] [6] on the one hand and Martinec [8] and Vafa and Warner [9] on the other that an alternative way of thinking about the same conformal fixed points of the heterotic string is via the theory ofexactly solvable models and their mean field theories. The virtue of the latter formulations lies in the fact that they are easier to deal with since they are formulated in a nongeometric fashion. They do, in particular, allow for a detailed analysis of the massless spectrum t which naturally falls in the same pattern as the Hodge diamond above when vacua with central charge nine are considered.

It is in these contexts that mirror symmetry was discovered. In [1] it was observed that for the majority of string vacua described by Landau-Ginzburg potentials with an isolated singularity there exists another vacuum for which the number of generations and antigenerations is reversed, or put differently, the Hodge diamond is flipped about the diagonal. Since the class of theories constructed in [1] can also be understood as a special class of Calabi-Yau manifolds this result immediately implies an explicit demonstration of mirror symmetry as an operation on the space of Calabi-Yau manifolds, a property of this space that came as quite a surprise to mathematicians.

Even though almost inconceivable, in principle the symmetry found in [1] might have been considered an accidental pairing of a priori independent theories. That this is not the case was shown in [2] via a construction involving orbifolding and fractional transformations. It was demonstrated in that paper that explicit mirror maps can indeed easily be established between many different types of Landau-Ginzburg theories with mirror spectra by mapping the orbifold mirror via fractional transformations of the order parameters onto a mirror Landau-Ginzburg potential, defining again a complete intersection Calabi-Yau manifold.

In [3] an alternative route to mirror symmetry was discovered in the process of constructing orbifolds of Gepner models. In this framework mirror symmetry can be described as a KramersWannier type symmetry of the underlying parafermionic theories.

One of the advantages of the construction of [2] is that it allows to mirror map the complete relevant moduli space [10] whereas the exactly solvable approach is restricted to one particular point in moduli space, at which the results of [2] of course recover the exactly solvable mirror theory.

The discovery of mirror symmetry per the constructions of [1] 2] 3] provided new impulse for the investigation of string compactification and a number of different avenues have been addressed

\footnotetext{
${ }^{1}$ In this article attention is focused on the generations and antigenerations. The gauge singlets are not considered.
} 
vigorously by many groups with two major focal points. On the one hand the question has been addressed [11, 12, 13, 14, 15, 16, 17, 18] whether mirror symmetry is an artefact of the particular framework in which it was discovered, and if not, then what the natural context is that extends the Landau-Ginzburg/Calabi-Yau framework. On the other hand the natural question arises what mirror symmetry can teach us about string theory. In a nutshell, mirror symmetry provides a tool that allows access to problems that before its discovery in the form of [1, 2, 3] were impossible to solve. The main foci in this direction are issues regarding the action of the modular group on moduli space [19, 20, 21, 22], questions concerning a mild type of topology change [23], and the computation of Yukawa couplings, pioneered in [19]. The latter has led to a breakthrough in mathematics and mirror symmetry is presently being used in this context as a hypothesis which leads to novel results in algebraic geometry. Whenever independent checks are possible, mirror symmetry has been shown to lead to correct results in all computations that have been performed so far for genus zero instantons [19, 24, 25, 26, 27, 28, 29, 30, 31, 32, 33, 34 as well as higher genus instantons [35, 36] in Calabi-Yau manifolds of complex dimension three, and for genus zero instantons in Brieskorn-Pham type Calabi-Yau manifolds for $\operatorname{dim}_{\mathbb{C}} M>3$ [37] 38].

The present paper is concerned with the first question - what is the proper framework for mirror symmetry? As far as sheer numbers are concerned it appears that the space of CalabiYau manifolds is the structure to go for because, very roughly, there are more Landau-Ginzburg models known than there are exactly solvable models and it is clear that many more Calabi-Yau manifolds can be constructed than there are Landau-Ginzburg theories. However, the space of all Calabi-Yau manifolds has a disconcerting property: it contains spaces which are rigid, i.e. they do not have string modes corresponding to complex deformations of the manifold, fields that describe generations in the low energy theory. Since mirror symmetry exchanges complex deformations and Kähler deformations of a manifold, the latter describing the antigenerations seen by a fourdimensional observer, it would seem that the mirror of a rigid Calabi-Yau manifold cannot be Kähler and hence does not describe a consistent string vacuum. In fact, it appears that the mirror vacuum cannot even be $N=1$ spacetime supersymmetric [39]. It follows then that the class of Calabi-Yau manifolds is not the appropriate setting by a long shot in which to discuss mirror symmetry and the question arises what the proper framework might be.

In this article I continue an investigation, initiated in [18, of a class of manifolds which generalizes the class of Calabi-Yau spaces of complex dimension $D_{\text {crit }}$ in a natural way. The manifolds involved are of complex dimension $\left(D_{\text {crit }}+2(Q-1)\right), Q>1$, and feature a positive first Chern class that is quantized in a particular way. Thus they do not describe, a priori, consistent 
string groundstates. Surprisingly however, it is possible to derive from these higher dimensional manifolds the spectrum of critical string vacua. This can be done not only for the generations but also for the antigenerations. Moreover a construction exists which for particular types of these new manifolds allows the derivation of the corresponding $D_{\text {crit }}$-dimensional Calabi-Yau manifold directly from the $\left(D_{\text {crit }}+2(Q-1)\right)$-dimensional space.

This new class of manifolds is, however, not in one to one correspondence with the class of Calabi-Yau manifolds as it contains manifolds which describe string vacua that do not contain massless modes corresponding to antigenerations. It is precisely this new type of manifold that is needed in order to construct mirrors of rigid Calabi-Yau manifolds without generations.

Hence the class of special Fano manifolds appears as a natural generalization of the standard Calabi-Yau framework. Further evidence that important Calabi-Yau constructions admit generalizations to special Fano manifolds has been presented in two recent papers [40, 41]. It has been shown in [40] that the computation of Yukawa couplings along the lines of [19] can be applied in this framework as well, whereas Batyrev and Borisov 41] present an interesting formulation of our construction in the framework of toric geometry. These authors generalize Batyrev's CalabiYau mirror construction [42] and do find, as conjectured in [18], a combinatorical understanding of mirror manifolds of Calabi-Yau manifolds of codimension larger than one by utilizing special Fano manifolds.

The paper is organized as follows: in Section 2 the manifolds in question will be briefly reviewed and their salient defining properties described. In Section 3 it will be shown how the spectrum of critical string vacua can be derived from the special class of Fano manifolds of Section 2. It will also be shown that for a number of different classes it is possible to derive the critical $D_{\text {crit }}{ }^{-}$ dimensional Calabi-Yau manifold directly from higher dimensional Fano manifolds. In Section 4 the geometrical construction of Section 3 will be generalized to arbitrary critical dimensions. Section 5 contains a more systematic discussion of the higher dimensional cohomology and Section 6 is devoted to an important 'degeneration phenomenon'. Sections 7 and 8 contain a discussion of the consequences for the present class of manifolds of the splitting and contraction construction of 443 as well as the topological identities that were used in 43 for the analysis of the class of complete intersection Calabi-Yau manifolds. In Section 9 it is shown how the geometrical construction of Section 3 pertains to the standard discussion of the Landau-Ginzburg/CalabiYau connection and Section 10 is devoted to the discussion of mirrors of rigid Calabi-Yau spaces. Two final Sections contain brief remarks regarding toric considerations and the generalization of the hypersurfaces of Section 2 to weighted complete intersections. 


\section{A Special Class of Fano Manifolds with Quantized Pos- itive First Chern Class}

The construction of noncritical manifolds proceeds via the following prescription [18]:

- Fix the central charge $c$ of the $(2,2)$-vacuum states and its critical dimension

$$
D_{\text {crit }}=c / 3 \text {. }
$$

- Choose a positive integer $Q \in \mathbb{N}$.

- Introduce $\left(D_{\text {crit }}+2 Q\right)$ complex coordinates $\left(z_{1}, \ldots, z_{D_{\text {crit }}+2 Q}\right), z_{i} \in \mathbb{C}$.

- Define an equivalence relation

$$
\left(z_{1}, \ldots, z_{D_{\text {crit }}+2 Q}\right) \sim\left(\lambda^{k_{1}} z_{1}, \ldots, \lambda^{k_{D_{c r i t}+2 Q}} z_{D_{\text {crit }}+2 Q}\right)
$$

where $\lambda \in \mathbb{C}^{*}$ is a nonzero complex number and the positive integers $k_{i} \in \mathbb{N}$ are the weights of these coordinates. The set of these equivalence classes defines so-called weighted projective spaces, compact manifolds which will be denoted by $\mathbb{P}_{\left(k_{1}, \ldots, k_{D_{c r i t}+2 Q}\right)}$.

- Define hypersurfaces in the ambient weighted projective space by imposing a constraint defined by polynomials $p$ of degree

$$
d=\frac{1}{Q} \sum_{i} k_{i}
$$

i.e. $p\left(\lambda^{i} z_{i}\right)=\lambda^{d} p\left(z_{i}\right)$.

The family of hypersurfaces embedded in the ambient space as the zero locus of $p$ will be denoted by

$$
\begin{aligned}
M_{D_{\text {crit }}+2(Q-1)} & =\left\{p\left(z_{1}, \ldots, z_{D_{\text {crit }}+2 Q}\right)=0\right\} \cap \mathbb{P}_{\left(k_{1}, \ldots, k_{\left.D_{\text {crit }}+2 Q\right)}\right.} \\
& =\mathbb{P}_{\left(k_{1}, \ldots, k_{D_{\text {crit }}+2 Q}\right)}\left[\frac{1}{Q} \sum_{i=1}^{D_{\text {crit }}+2 Q} k_{i}\right]
\end{aligned}
$$

and will be called a configuration. 
Relation (2.3) is the defining property of the class of spaces to be considered below. It is a rather restrictive condition in that it excludes many types of varieties which are transverse and even smooth but are not of physical relevance 9 . A simple example is the Fermat type hypersurface

$$
\mathbb{P}_{(420,280,210,168,140,120,105)}[840] \ni\left\{p=\sum_{i=1}^{7} z_{i}^{i+1}=0\right\}
$$

which is not only quasihomogeneous but also transverse, i.e. a quasismooth manifold. The fact that this quasismooth variety is rather different from the manifolds of type (2.4) can be understood in a more geometrical way. Namely, what makes the Fano varieties of type (2.4) special is, among other things, a property that they share with Calabi-Yau manifolds: they do not contain singular sets of codimension one. For Calabi-Yau spaces this means that the singular sets are either points or (complex) curves, but never surfaces. In a more general context this fact translates into the statement that the only resolutions that have be performed are so-called small resolutions. The above Fermat type hypersurface (2.5) on the other hand contains the singular codimension-1 four-fold $S=\mathbb{P}_{(210,140,105,84,70,60)}[420]$.

Alternatively, Fano manifolds of the special type (2.4) above may be characterized via a curvature constraint. Because of (2.3) the first Chern class is given by

$$
c_{1}\left(M_{D_{c r i t}+2(Q-1)}\right)=(Q-1) c_{1}(\mathcal{N})
$$

where $c_{1}(\mathcal{N})=d h$ is the first Chern class of the normal bundle $\mathcal{N}$ of the hypersurface $M_{D_{\text {crit }}+2(Q-1)}$ and $h$ is the pullback of the Kähler form $H \in \mathrm{H}^{(1,1)}\left(\mathbb{P}_{\left(k_{1}, \ldots, k_{D_{c r i t}+2 Q}\right)}\right)$ of the ambient space. Hence the first Chern class is quantized in multiples of the degree of the hypersurface $M_{D_{c r i t}+2(Q-1)}$. For $Q=1$ the first Chern class vanishes and the manifolds for which (2.3) holds are Calabi-Yau manifolds, defining consistent groundstates of the supersymmetric closed string. For $Q>1$ the first Chern class is nonvanishing and therefore these manifolds cannot possibly describe vacua of the critical string, or so it seems.

It has been shown in [18] however that it is possible to derive from these higher dimensional manifolds the massless spectrum of critical vacua. It is furthermore possible, for certain subclasses of hypersurfaces of type (2.4), to construct Calabi-Yau manifolds $M_{C Y}$ of dimension $D_{\text {crit }}$ and complex codimension

$$
\operatorname{codim}_{\mathbb{C}}\left(M_{C Y}\right)=Q
$$

\footnotetext{
${ }^{2}$ It will become clear below that this definition is rather natural in the context of the theory of Landau-Ginzburg string vacua with an arbitrary number of scaling fields. A particular simple manifold in this class, the cubic sevenfold $\mathbb{P}_{8}[3]$, has been the subject of refs. 40] 44 .
} 
directly from these manifolds. The integer $Q$ thus plays a central rôle: the critical dimension is the dimension of the noncritical manifolds offset by twice the coefficient of the first Chern class of the normal bundle of the hypersurface, which involves $Q$. The physical interpretation of the integer $Q$ is that of a total charge associated to the corresponding Landau-Ginzburg theory which determines the codimension of the Calabi-Yau manifold which it describes.

As mentioned already in the introduction the class of spaces defined by (2.4) contains manifolds with no antigenerations and hence it is necessary to have some way other than Calabi-Yau manifolds to represent string groundstates if one intends to understand the relation of the latter to higher dimensional special Fano varieties. One possible way to do this is to relate them to Landau-Ginzburg theories: any manifold of type (2.4) can be viewed as a projectivization of an affine noncompact hypersurface

$$
\mathbb{C}_{\left(k_{1}, \ldots, k_{N+2}\right)}[d] \ni\left\{p\left(z_{1}, \ldots, z_{N+2}\right)=0\right\}
$$

via a weighted equivalence relation

$$
\left(z_{1}, \ldots, z_{N+2}\right) \equiv\left(\lambda^{k_{1}} z_{1}, \ldots, \lambda^{k_{N+2}} z_{N+2}\right)
$$

Because the polynomial $p$ is assumed to be transverse in the projective ambient space the affine variety has a very mild singularity: it has an isolated singularity at the origin defining what is called a catastrophe in the mathematical literature. The polynomial ring of such a catastrophe is isomorphic to the chiral ring of a $\mathrm{N}=2$ supersymmetric Landau-Ginzburg theory and hence allows us to compute physical quantities via methods in this framework 5 .

Even though the manifolds (2.4) therefore correspond to LG theories of central charge $c=$ $3 D_{\text {crit }}$ they can, however, not be identical to such theories: Consider the case when the critical dimension of the internal space corresponds to our world, i.e. $D_{\text {crit }}=3$ and $Q=2$. The cohomology of $M_{5}$ leads to the Hodge diamond

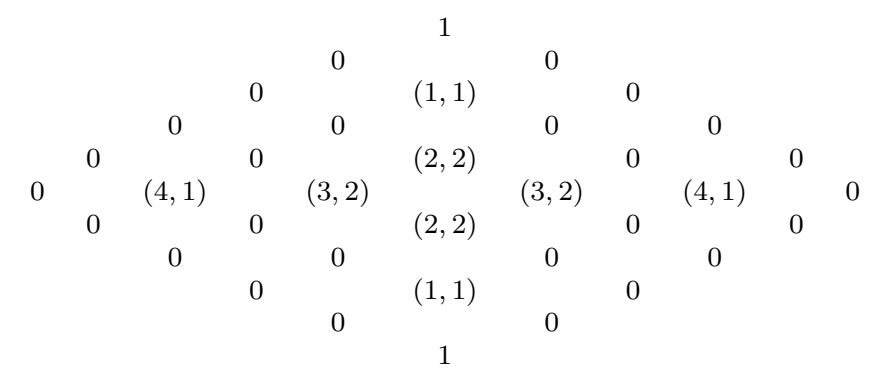

\footnotetext{
${ }^{3}$ Landau-Ginzburg theories are discussed in greater detail in a different context in Section 9.
} 
where $(p, q)$ denotes the dimension $h^{(p, q)}$ of the cohomology group $\mathrm{H}^{(p, q)}\left(M_{5}\right)$.

It is clear from this Hodge diamond that the higher dimensional manifolds will contain more modes than the critical vacuum and hence the relation of the spectrum of the critical vacuum and the cohomology of the noncritical manifolds will be a nontrivial one. It is this question which is the subject of the next Section.

\section{Special Fano Varieties and Critical String Vacua: the Physical Dimension}

Even though for $Q>1$ the spaces (2.4) clearly are not of Calabi-Yau type and hence do not, a priori, lead to consistent string vacua, they do in fact encode essential information about these string vacua. Most importantly it is possible to derive from these higher dimensional manifolds the massless spectrum of critical vacua. Furthermore we will see that for certain subclasses of hypersurfaces of type (2.4) it is possible to construct Calabi-Yau manifolds $M_{C Y}$ of dimension $D_{\text {crit }}$ and complex codimension $\operatorname{codim}_{\mathbb{C}}\left(M_{C Y}\right)=Q$ directly from these manifolds.

A first step in the analysis of any physical theory is to determine its physical spectrum; hence the first question to address is how it is possible to derive the massless spectrum of the corresponding string vacua from these higher dimensional manifolds. In order to be able to formulate our geometrical framework in a concise way it is useful to recall some of the salient characteristics of hypersurfaces in weighted projective spaces. The feature most relevant for the following considerations comes from the fact that weighted projective spaces are singular objects, the singularities being of orbifold type. Submanifolds defined via some polynomial constraints will, in general, intersect these singularities and thus be singular themselves. Such singular spaces, called $\mathrm{V}$-manifolds in older references, so far have not been of interest for string physics, rather it is the smoothed out, 'resolved', manifold which has been the physically important object. Such is the case for the critical situation $Q=1$.

For $Q>1$ it is clear that a projection is needed which selects from the cohomology of the Fano variety the physically relevant part. In the following it will be shown that the following selection rule provides for a geometrical projection of the Hodge diamond of the special Fano manifold. 
The generation and antigeneration spectrum of a $c=D_{\text {crit }}$ vacuum of the $N=1$ spacetime supersymmetric string is parametrized by the cohomology carried by the singular subvarieties of the Fano fold together with the monomials of the integrally charged subring of the polynomial ring.

It is important to realize that our prescription is radically different from the $Q=1$ case where not only the cohomology carried by the singular sets is important but also the cohomology induced by the fibers of the bundles that are introduced in the process of resolving these singularities.

Of immediate concern, of course, are vacua which are mirrors of rigid Calabi-Yau manifolds: Consider the orbifold $T_{1}^{3} / \mathbb{Z}_{3}^{2}$ where the two actions are defined as $\left(z_{1}, z_{4}\right) \longrightarrow\left(\alpha z_{1}, \alpha^{2} z_{4}\right)$, all other coordinates invariant and $\left(z_{1}, z_{7}\right) \longrightarrow\left(\alpha z_{1}, \alpha^{2} z_{7}\right)$, all other invariant. Here $\alpha$ is the third root of unity. The resolution of the singular orbifold leads to a Calabi-Yau manifold with 84 antigenerations and no generations [45]. This is precisely the mirror flipped spectrum of the exactly solvable tensor model $1^{9}$ of 9 copies of $N=2$ superconformal minimal models at level $k=1$ [5] which can be described in terms of the Landau-Ginzburg potential $W=\sum z_{i}^{3}$ which belongs to the configuration $\mathbb{C}_{(1,1,1,1,1,1,1,1,1)}[3]$. The generations of this theory are obtained by counting the number of monomials of charge unity, of which there are 84. Imposing the GSO projection by modding out a $\mathbb{Z}_{3}$ symmetry this Landau-Ginzburg theory does not lead to new generations and furthermore does not create antigenerations. Thus this LG theory leads to the same spectrum as the $1^{9}$ model 5 .

This Landau-Ginzburg theory clearly is a mirror candidate for the resolved torus orbifold just mentioned 40, 44 and the question arises whether a manifold corresponding to this LG potential can be found. Since the theory does not contain modes corresponding to $(1,1)$-forms it appears that the manifold cannot be Kähler and hence not projective. Thus it appears that the 7-dimensional manifold $\mathbb{P}_{8}[3]$ whose polynomial ring is identical to the chiral ring of the LG theory is merely useful as an auxiliary device in order to describe one sector of the critical LG string vacuum.

There exists one further theory in the space of all Landau-Ginzburg vacua which does not have any antigenerations and which is distinct from the one discussed above. Like the previous

\footnotetext{
${ }^{3}$ It would seem that an obvious generalization of this 7 -dimensional smooth manifold is the infinite class of models $\mathbb{C}_{(1,1,1,1,1,1,1,1,1+3 p)}[3+p]$, but since the manifolds $(2.4)$ are required to be transverse the only possibility is $p=0$.
} 
example it originates from an exactly solvable model in the class of Gepner models

$$
\left(2^{6}\right)_{A^{6}}^{(0,90)} \equiv \mathbb{C}_{(1,1,1,1,1,1,2)}^{*}[4] \ni\left\{\sum_{i} z_{i}^{4}+z_{7}^{2}=0\right\}
$$

and leads to an obviously smooth manifold $\mathbb{P}_{(1,1,1,1,1,1,2)}[4]$ as well.

Our geometrical projection immediately explains why mirrors of rigid vacua are so different: in the context of special Fano manifolds they are distinguished by the fact that they do not lead to Kähler modes because they are described by smooth spaces. Thus these two theories fit in very well in our way of thinking about the critical spectrum of the corresponding string vacuum.

Vacua without antigenerations are rather exceptional however; the generic groundstate will have both sectors, generations and antigenerations. The idea described above to derive the spectrum works for higher dimensional manifolds corresponding to different types of critical vacua, as will be shown now.

To be concrete consider the exactly solvable tensor theory $\left(1 \cdot 16^{3}\right)_{A_{2} \otimes E_{7}^{3}}$ with 35 generations and 8 antigenerations which corresponds to a Landau-Ginzburg theory belonging to the configuration

$$
\mathbb{C}_{(2,3,2,3,2,3,3)}^{*}[9]^{(8,35)}
$$

and which induces, via projectivization, a 5-dimensional weighted hypersurface

$$
\mathbb{P}_{(2,2,2,3,3,3,3)}[9] \ni\left\{p=\sum_{i=1}^{3}\left(y_{i}^{3} x_{i}+x_{i}^{3}\right)+x_{4}^{3}=0\right\},
$$

which contains the orbifold singularity sets

$$
\begin{array}{ll}
\mathbb{Z}_{3}: & \mathbb{P}_{3}[3] \ni\left\{p_{1}=\sum_{i=1}^{4} x_{i}^{3}=0\right\}, \\
\mathbb{Z}_{2}: & \mathbb{P}_{2} .
\end{array}
$$

The $\mathbb{Z}_{3}$-singular set is a smooth cubic surface which supports seven $(1,1)$-forms which follows from $\chi\left(\mathbb{P}_{3}[3]\right)=9$ and the fact that $c_{1}\left(\mathbb{P}_{3}[3]\right)>0$. The $\mathbb{Z}_{2}$ singular set is just the projective plane and therefore adds one further $(1,1)$-form. Hence the singularities induced on the 5 -fold by the singularities of the ambient weighted projective space $\mathbb{P}_{(2,2,2,3,3,3,3)}$ give rise to a total of eight $(1,1)$-forms. A simple count leads to the result that the subring of monomials of charge unity is of dimension 35. Thus we have derived the spectrum of the critical theory from the noncritical manifold (3.3). 
The singularity structure (3.4) of the manifold under consideration is rather intriguing and suggests that the idea introduced above of relating the spectrum of the string vacuum to the singularity structure of the Fano variety implies much more: namely it leads also to a prescription of how to derive from these higher dimensional manifolds the Calabi-Yau manifold of critical dimension! Thus a canonical prescription is obtained which also allows to pass from the LandauGinzburg theory to its geometrical counterpart.

This works as follows: Recall that the structure of the singularities of the weighted hypersurface only involved part of the superpotential, namely the cubic polynomial $p_{1}$ which determined the $\mathbb{Z}_{3}$ singular set described by a surface. The superpotential thus splits naturally into the two parts

$$
p=p_{1}+p_{2}
$$

where $p_{2}$ is the remaining part of the polynomial. The idea now is to consider the product $\mathbb{P}_{3}[3] \times \mathbb{P}_{2}$, where the factors are determined by the singular sets of the higher dimensional space, and to impose on this 4-dimensional space a constraint described by the remaining part of the polynomial which did not take part in the definition of the singularities of the special Fano 5 -fold. In the case at hand this leaves a polynomial of bidegree $(3,1)$ and hence we are lead to a manifold embedded in the configuration

$$
\begin{aligned}
& \mathbb{P}_{2} \\
& \mathbb{P}_{3}
\end{aligned}\left[\begin{array}{ll}
3 & 0 \\
1 & 3
\end{array}\right]
$$

via the polynomials

$$
\begin{aligned}
& p_{1}=y_{1}^{3} x_{1}+y_{2}^{3} x_{2}+y_{3}^{3} x_{3}, \\
& p_{2}=\sum_{i=1}^{4} x_{i}^{3} .
\end{aligned}
$$

This is precisely the Calabi-Yau 3-fold constructed in [46], the exactly solvable model of which was later found in [7]. Thus we have found how to construct from the noncritical manifold (3.3) a critical Calabi-Yau manifold of the correct physical dimension.

A class of manifolds of a different type which can be discussed in this framework rather naturally is defined by

$$
\mathbb{P}_{\left(2 k, K-k, 2 k, K-k, 2 k_{3}, 2 k_{4}, 2 k_{5}\right)}[2 K]
$$

where $K=k+k_{3}+k_{4}+k_{5}$. Assume, for the moment, that $K / k$ and $K / k_{i}$ are integers. The potentials can then be chosen to be of the form

$$
W=\sum_{i=1}^{2}\left(x_{i}^{K / k}+x_{i} y_{i}^{2}\right)+x_{3}^{K / k_{3}}+x_{4}^{K / k_{4}}+x_{5}^{K / k_{5}} .
$$


and the singularities of these manifolds are

$$
\begin{aligned}
\mathbb{Z}_{2}: & \mathbb{P}_{\left(k, k, k_{3}, k_{4}, k_{5}\right)}[K] \ni\left\{\sum_{i} x_{i}^{K / k_{i}}=0\right\}, \\
\mathbb{Z}_{K-k}: & \mathbb{P}_{1} .
\end{aligned}
$$

This suggests that there exists a canonical way to proceed to the corresponding Calabi-Yau manifold of these theories by considering manifolds embedded in

$$
\mathbb{P}_{1} \mathbb{P}_{\left(k, k, k_{3}, k_{4}, k_{5}\right)}\left[\begin{array}{cc}
2 & 0 \\
k & K
\end{array}\right]
$$

defined by polynomials

$$
\begin{aligned}
& p_{1}=y_{1}^{2} x_{1}+y_{2}^{2} x_{2} \\
& p_{2}=x_{1}^{K / k}+x_{2}^{K / k}+x_{3}^{K / k_{3}}+x_{4}^{K / k_{4}}+x_{5}^{K / k_{5}} .
\end{aligned}
$$

That this correspondence is in fact correct, can be inferred from the work of [47] where it was shown that these codimension-2 weighted complete intersection Calabi-Yau manifolds correspond to $N=2$ minimal exactly solvable tensor models of the type

$$
\left[2\left(\frac{K}{k}-1\right)\right]_{D}^{2} \otimes \prod_{i=3}^{5}\left(\frac{K}{k_{i}}-2\right)_{A} .
$$

where the subscripts indicate the type of the affine invariants chosen for the individual levels.

The constraints that $k$ and the $k_{i}$ divide $K$ have been imposed purely for convenience since it allows for a nice coherent description of the resulting class. These restrictions however can be relaxed to more general types of potentials, the discussion of which will take up the remainder of this Section. The types include, among others:

- Single tadpole involving, say, the variables $x_{4}, x_{5}$

$$
x_{4}^{a}+x_{4} x_{5}^{b}
$$

which leads to the modified divisibility criterion $\frac{K}{k_{4}}, \frac{1}{k_{5}}\left(K-k_{4}\right) \in \mathbb{N}$. An example is furnished by

$$
\mathbb{P}_{(7,2,7,2,4,4,6)}[16] \ni\left\{p=\sum_{i=1}^{2}\left(x_{i}^{2} y_{i}+y_{i}^{8}\right)+y_{3}^{4}+y_{4}^{4}+y_{4} y_{5}^{2}=0\right\}
$$

which leads to

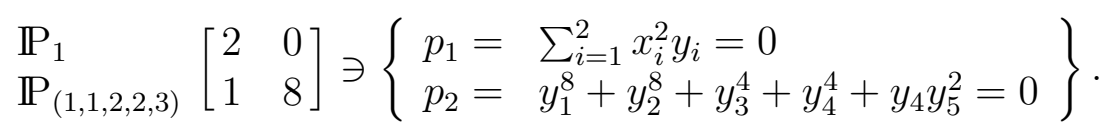




\section{- Isolated double tadpole}

$$
x_{3}^{a}+x_{3} x_{4}^{b}+x_{4} x_{5}^{c}
$$

involving three coordinates for which $\frac{K}{k_{3}}, \frac{1}{k_{4}}\left(K-k_{3}\right), \frac{1}{k_{5}}\left(K-k_{4}\right) \in \mathbb{N}$. An example of this type is obtained via

$$
\mathbb{P}_{(17,2,17,2,8,12,14)}[36] \ni\left\{p=\sum_{i=1}^{2}\left(x_{i}^{2} y_{i}+y_{i}^{18}\right)+y_{3}^{3} y_{4}+y_{4}^{3}+y_{3} y_{5}^{2}=0\right\}
$$

from which our construction derives the Calabi-Yau 3-fold

$$
\begin{aligned}
& \mathbb{P}_{1} \\
& \mathbb{P}_{(1,1,4,6,7)}
\end{aligned}\left[\begin{array}{cc}
2 & 0 \\
1 & 18
\end{array}\right] \ni\left\{\begin{array}{l}
p_{1}=\sum_{i=1}^{2} x_{i}^{2} y_{i}=0 \\
p_{2}=y_{1}^{18}+y_{2}^{18}+y_{3}^{3} y_{4}+y_{4}^{3}+y_{3} y_{5}^{2}=0
\end{array}\right\}
$$

- Nonisolated tadpoles. It is not necessary however to have the variables $x_{3}, x_{4}, x_{5}$ completely decoupled from the rest of the coordinates. Examples abound where they are linked nontrivial ways to other variables as well. This is illustrated by the 5 -fold

$$
\mathbb{P}_{(3,4,2,3,4,2,2)}[10] \ni\left\{p=\sum_{i=1}^{2}\left(x_{i}^{2} y_{i}+y_{i}^{2} z_{i}+z_{i}^{5}\right)+z_{3}^{5}=0\right\}
$$

from which we can derive the Calabi-Yau 3-fold

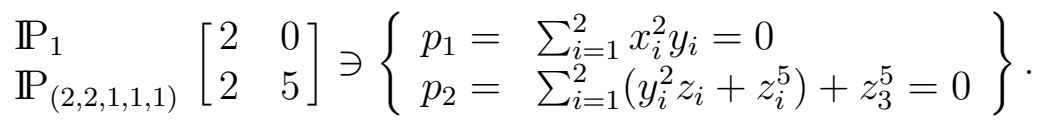

- Exceptional types. Finally, it should be emphasized that our construction also works for polynomials which contain so-called exceptional terms, an example in point being

$$
\mathbb{P}_{(2,4,5,2,4,5,6)}[14] \ni\left\{p=\sum_{i=1}^{2}\left(x_{i}^{2} y_{i}+y_{i}^{3} z_{i}+z_{i}^{7}\right)+z_{1} u+u y_{1}^{2}=0\right\}
$$

leading to the Calabi-Yau 3-fold

$$
\begin{aligned}
& \mathbb{P}_{1} \\
& \mathbb{P}_{(2,2,1,1,3)}
\end{aligned}\left[\begin{array}{ll}
2 & 0 \\
2 & 7
\end{array}\right] \ni\left\{\begin{array}{l}
p_{1}=\sum_{i=1}^{2} x_{i}^{2} y_{i}=0 \\
p_{2}=\sum_{i=1}^{2}\left(y_{i}^{3} z_{i}+z_{i}^{7}\right)+z_{1} u+u y_{1}^{2}=0
\end{array}\right\}
$$

It is clear from this discussion that the spaces described by (3.11) describe a much wider class of models than the known exactly solvable spaces which were the focus of ref. 477.

The picture that emerges from these constructions then is the following: embedded in the higher dimensional manifold is a submanifold which is fibered. The base and the fibers of this 
fibration are determined by the singular sets of the ambient manifold. The Calabi-Yau manifold itself is a hypersurface embedded in this fibered submanifold. A heuristic sketch of the geometry is shown in the Figure 1.

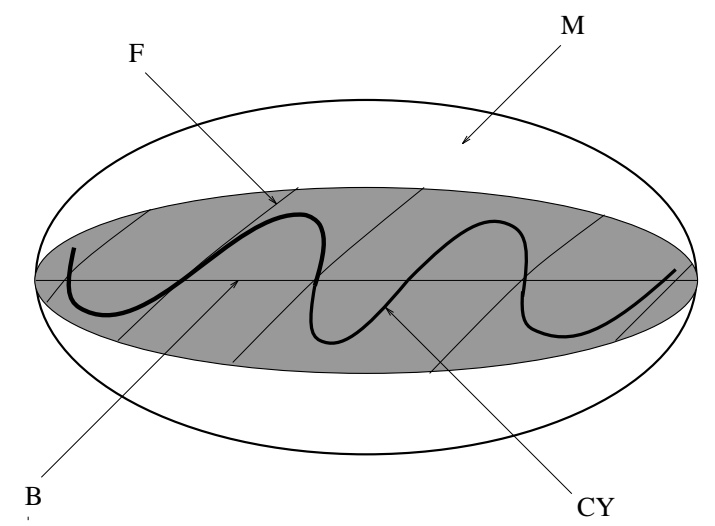

Figure 1: The Calabi-Yau manifold $C Y$ is embedded in the fibered submanifold $B \times F$ of the higher dimensional manifold $M$. Both the base $B$ and the fibers $F$ of the bundle (indicated by the shaded surface) are determined by the singular sets of the manifold $M$.

The examples above illustrate the simplest situation that can appear. In more complicated manifolds the singularity structure will consist of hypersurfaces whose fibers and/or base themselves are fibered, leading to an iterative procedure. Figure 2 depicts more general situations of such a type.

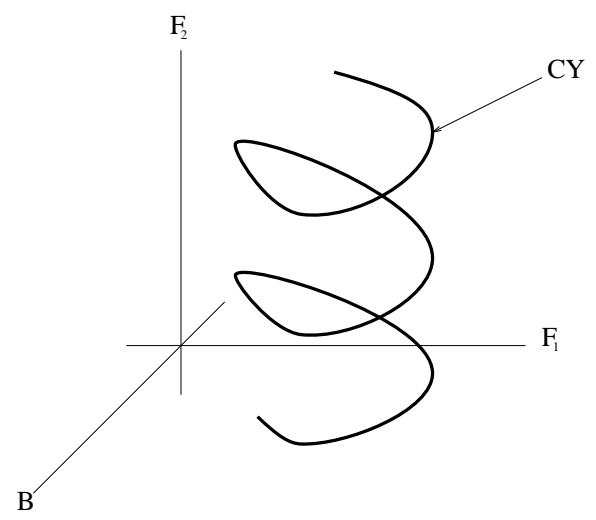

Figure 2: The Calabi-Yau manifold $C Y$ is embedded in the fibered submanifold $B \times F_{1} \times \cdots \times F_{n}$ determined by the singular sets of the higher dimensional manifold $M$.

The fibered submanifold of the higher dimensional Fano variety will, in those cases, be of codimension larger than one and the Calabi-Yau manifold will be described by a submanifold 
with codimension larger than one as well. To illustrate this point consider the 9-fold

$$
\mathbb{P}_{(5,5,6,6,6,4,4,4,8,8,8)}[16] \ni\left\{\sum_{i=1}^{2}\left(u_{i}^{2} v_{i}+v_{i}^{2} w_{i}+w_{i}^{2} x_{i}+x_{i}^{2}\right)+v_{3}^{2} w_{3}+w_{3}^{2} x_{3}+x_{3}^{2}=0\right\} .
$$

The $\mathbb{Z}_{2}$-fibering leads to the split

$$
\mathbb{P}_{1} \mathbb{P}_{(3,3,3,2,2,2,4,4,4)}\left[\begin{array}{cc}
2 & 0 \\
3 & 16
\end{array}\right]
$$

which in turn leads to the $\mathbb{Z}_{2}$ split

$$
\begin{aligned}
& \mathbb{P}_{1} \\
& \mathbb{P}_{2} \\
& \mathbb{P}_{(1,1,1,2,2,2)}
\end{aligned} \quad\left[\begin{array}{lll}
2 & 0 & 0 \\
1 & 2 & 0 \\
0 & 1 & 4
\end{array}\right]
$$

which finally leads to

$$
\begin{aligned}
& \mathbb{P}_{1} \\
& \mathbb{P}_{2} \\
& \mathbb{P}_{2} \\
& \mathbb{P}_{2}
\end{aligned}\left[\begin{array}{llll}
2 & 0 & 0 & 0 \\
1 & 2 & 0 & 0 \\
0 & 1 & 2 & 0 \\
0 & 0 & 1 & 2
\end{array}\right] \ni\left\{\begin{array}{l}
p_{1}=\sum u_{i}^{2} v_{i} \\
p_{2}=\sum v_{i}^{2} w_{i} \\
p_{3}=\sum w_{i}^{2} x_{i} \\
p_{4}=\sum x_{i}^{2}
\end{array}\right\}
$$

Thus the 9-fold fibers iteratively and the splits of the polynomial $p$ are dictated by the fibering.

A further manifold leading to a critical vacuum with codimension larger than two and which is of a type that will make another appearence later in Section 9 is the 7 -fold

$$
\mathbb{P}_{(1,1,6,6,2,2,2,2,2)}[8] \ni\left\{\sum_{i=1}^{2}\left(x_{i}^{2} y_{i}+y_{i} z_{i}+z_{i}^{4}\right)+z_{3}^{4}+z_{4}^{4}+z_{5}^{4}=0\right\}
$$

which leads to the $\mathbb{Z}_{2}$ fibering $\mathbb{P}_{1} \times \mathbb{P}_{(3,3,1,1,1,1,1)}[4]$ which in turn leads to the $\mathbb{Z}_{3}$ fibering $\mathbb{P}_{1} \times$ $\mathbb{P}_{1} \times \mathbb{P}_{4}[4]$ and finally to the Calabi-Yau configuration

$$
\begin{aligned}
& \mathbb{P}_{1} \\
& \mathbb{P}_{1} \\
& \mathbb{P}_{4}
\end{aligned}\left[\begin{array}{lll}
2 & 0 & 0 \\
1 & 1 & 0 \\
0 & 1 & 4
\end{array}\right] \ni\left\{\begin{array}{l}
p_{1}=\sum_{i=1}^{2} x_{i}^{2} y_{i}=0 \\
p_{2}=\sum_{i=1}^{2} y_{i} z_{i}=0 \\
p_{3}=\sum_{j=1}^{5} z_{i}^{4}=0
\end{array}\right\}
$$

which is of codimension 3. This example is of interest because it shows, as will become clear later, that there are nontrivial relations between the higher dimensional special Fano manifolds. This will follow from the processes of splitting and contraction of Calabi-Yau manifolds introduced in ref. 433. It will become obvious that in fact the Calabi-Yau manifold (3.29) is an ineffective split of a Calabi-Yau manifold in the class (3.8). Thus there also exists a corresponding relation between the higher dimensional manifolds. 


\section{Generalization to Arbitrary Critical Dimensions}

Even though the examples discussed in the previous Section were all concerned with 6-dimensional Calabi-Yau manifolds and the way they are embedded in the new class of special Fano spaces, the ideas introduced in [18] and described in greater detail above are not specific to this dimension. Instead of considering compactifications down to the physical dimension, namely four, one might contemplate compactifying down to two, six or eight dimensions, or else, discuss the class of manifolds (2.4) not in the context of compactification at all.

To illustrate this point consider the doubly infinite class of $(m+n)$-dimensional complex compact manifolds

$$
\mathbb{P}_{(m+1, n-1, m+1, n-1, \ldots, m+1, n-1, m+1, \cdots, m+1)}[(m+1) n]
$$

with $(m+1)$ pairs of coordinates with weights $(m+1, n-1)$ and $(n-m)$ coordinates of weight $(m+1)$, defined by polynomials

$$
p=\sum_{i=1}^{m+1}\left(x_{i}^{n}+x_{i} y_{i}^{m+1}\right)+\sum_{j=m+2}^{n+1} x_{j}^{n} .
$$

These $(m+n)$-dimensional spaces lead to $Q=2$ and hence define, according to our construction, Calabi-Yau manifolds of dimension $(m+n-2)$ embedded in the configurations

$$
\mathbb{P}_{m}\left[\begin{array}{cc}
(m+1) & 0 \\
1 & n
\end{array}\right]
$$

via the zero locus of the polynomials

$$
p_{1}=\sum_{i} y_{i}^{m+1} x_{i}, \quad p_{2}=\sum_{i=1}^{n+1} x_{i}^{n} .
$$

The simplest example is, of course, the case $n=2$ where the higher dimensional manifold is a 3-fold described by

$$
\mathbb{P}_{(2,1,2,1,2)}[4] \ni\left\{\sum_{i=1}^{2}\left(z_{i}^{2}+z_{i} y_{i}^{2}\right)+z_{3}^{2}=0\right\}
$$

with a $\mathbb{Z}_{2}$ singular set isomorphic to the sphere $\mathbb{P}_{2}[2] \sim \mathbb{P}_{1}$ which contributes one $(1,1)$-form. The singularity structure of the 3 -fold then relates this space to the complex torus described by the algebraic curve

$$
\begin{aligned}
& \mathbb{P}_{1} \\
& \mathbb{P}_{2}
\end{aligned}\left[\begin{array}{ll}
2 & 0 \\
1 & 2
\end{array}\right]
$$


The Landau-Ginzburg theory corresponding to this theory derives from an exactly solvable tensor model $\left(2^{2}\right)_{D^{2}}$ described by two $N=2$ superconformal minimal theories at level $k=2$ equipped with the affine D-invariant.

It is of interest to consider the cohomology groups of the 3 -fold itself. With the third Chern class $c_{3}=2 h^{3}$ the Euler number of the singular space is

$$
\chi_{s}=\int c_{3}=1
$$

and hence the Euler number of the resolved manifold is

$$
\tilde{\chi}=1-(2 / 2)+2 \cdot 2=4 .
$$

Since the singular set is a sphere its resolution contributes just one $(1,1)$-form and hence the second Betti number becomes $b_{2}=2$. With $\tilde{\chi}=2\left(1+h^{(1,1)}\right)-2 h^{(2,1)}$ it follows that $h^{(2,1)}=1$ and therefore the Hodge diamond becomes

$\begin{array}{cccccccc} & & & 1 & & & \\ & 0 & 0 & & 0 & & \\ 0 & & 1 & 2 & & 0 & \\ & 0 & & 2 & 1 & & 0\end{array}$.

The case $n=3$ is exceptional because it leads to weights that are all equal and hence involves a higher dimensional manifold $\mathbb{P}_{5}[3]$ that is smooth and hence the naive expectation is that the construction breaks down. This, however, is not the case, the observation being that the polynomial to be considered is not of Fermat type and indeed has a $\mathbb{Z}_{2}$ symmetry. Instead of considering the smooth manifold $\mathbb{P}_{5}[3]$ the space to use instead then is the orbifold $\mathbb{P}_{5}[3] / \mathbb{Z}_{2}$ and one needs to compare the cohomology groups of this space with the spectrum of K3

$$
K 3=\frac{\mathbb{P}_{1}}{\mathbb{P}_{3}}\left[\begin{array}{ll}
2 & 0 \\
1 & 3
\end{array}\right],
$$

which consists of $h^{(0,0)}=h^{(2,2)}=h^{(2,0)}=h^{(0,2)}=1$ and $h^{(1,1)}=20$, all other Hodge numbers are zero. Hence the Euler number becomes $\chi(K 3)=24$. The Euler number for the noncritical manifold

$$
\mathbb{P}_{5}[3]
$$

is easily computed to be $\chi=27$. Since the manifold is Kähler, $h^{(p, q)}=h^{(q, p)}$ and because of Poincaré duality, $b_{p}=b_{8-p}$. Because the manifold has positive first Chern class, it follows from 
Kodaira's vanishing theorem that $h^{(p, 0)}=0$ for $p \neq 0$ and via Lefshetz's hyperplane theorem it is known that below the middle dimension all the cohomology is inherited from the ambient space and therefore the only nonvanishing cohomology groups lead to $h^{(0,0)}=h^{(1,1)}=1$. It can be shown that $h^{(3,1)}=h^{(1,2)}=1$ and therefore the only remaining cohomology is in $H^{(2,2)}$. Since

$$
\chi=2\left(b_{0}+b_{2}\right)+2+h^{(2,2)}=6+h^{(2,2)},
$$

it follows that $h^{(2,2)}=21=20+1$. The $\mathbb{Z}_{2}$ orbifold of this space introduces one further $(1,1)$-form and hence we obtain the spectrum of K3 plus one additional mode. Thus the Hodge diamond splits as

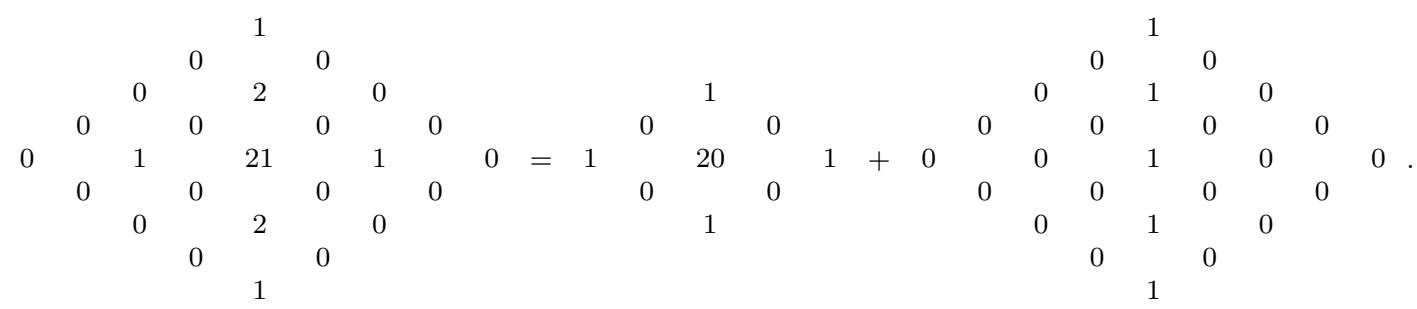

This example is also useful because it indicates a generalization of the considerations of the previous Section. The surprising new feature of this manifold is that even though the higher dimensional manifold did not have any orbifold singularities it was nevertheless possible to decompose it in such a way as to construct a Calabi-Yau manifold from it. This was possible because the defining equation was not of Fermat type but involved coupling between the fields. Because of this the manifold featured a new $\mathbb{Z}_{2}$ symmetry not present in the Fermat hypersurface and it is this new symmetry that dictated how to perform the decomposition. This indicates that even for smooth higher dimensional manifolds it is possible to relate them to Calabi-Yau manifolds once one moves away from the symmetric point.

Similar situations appear whenever

$$
n=m+2
$$

in which case the defining equations in the ordinary projective space are not of Fermat type and feature an additional $\mathbb{Z}_{m+1}$ symmetry that is not induced by the ambient space.

The series (4.3) can be generalized to weighted critical manifolds as is illustrated by the following polynomial

$$
p=\sum_{i=1}^{3}\left(x_{i}^{3}+x_{i} y_{i}^{3}\right)+\sum_{j=4}^{5} x_{i}^{6}
$$


which originates from the tensor model $\left(16^{3} \cdot 4\right)_{E^{3} \otimes A^{2}}$ with central charge $c=12$ and whose zero locus belongs to the configuration

$$
\mathbb{P}_{(6,4,6,4,6,4,3,3)}[18]
$$

The critical manifold derived from this 6-fold belongs to the 4-dimensional configuration class

$$
\mathbb{P}_{(2,2,2,1,1)}\left[\begin{array}{ll}
3 & 0 \\
2 & 6
\end{array}\right]
$$

which is indeed a Calabi-Yau deformation class.

Another infinite class of Calabi-Yau manifolds which are contained in higher dimensional spaces of the type (2.4) is furnished by the series of manifolds

$$
\mathbb{P}_{n}[n+1], \quad n \in \mathbb{N}
$$

which is related to the set of $(n+1)$-folds

$$
\begin{array}{rll}
\mathbb{P}_{\left(1,1, \ldots, 1, \frac{n+1}{2}, \frac{n+1}{2}\right)}[n+1], & (n+1) & \text { even }, \\
\mathbb{P}_{(2,2, \ldots, 2, n+1, n+1)}[2(n+1)], & (n+1) & \text { odd } .
\end{array}
$$

For $(n+1)$ even the $\mathbb{Z}_{\frac{n+1}{2}}$-singularity set consists of two points and therefore one obtains two copies of the Calabi-Yau $\mathbb{P}_{n}[n+1]$ and for odd $(n+1)$ the $\mathbb{Z}_{n+1}$ singular set is again two points and the $\mathbb{Z}_{2}$ singular set is the Calabi-Yau.

The simplest case is $n=2$ for which the resolution of the orbifold singularities of the noncritical 3-fold

$$
\mathbb{P}_{(2,2,2,3,3)}[6]
$$

leads to two independent Hodge numbers $h^{(1,1)}=4, h^{(2,1)}=2$ and hence the Hodge diamond contains twice the Hodge diamond of the torus, as it must, according to the geometrical picture described above. Similarly $\mathbb{P}_{(2,2,2,2,2,5,5)}[10]$ leads to two copies of the critical quintic.

Our construction is not restricted to the infinite series defined in (4.3) or its weighted generalization as the next example illustrates. A five-dimensional critical vacuum of higher codimension is obtained by considering the Landau-Ginzburg potential

$$
W=\sum_{j=1}^{2}\left(u_{i}^{3}+u_{i} v_{i}^{2}\right)+\sum_{i=3}^{5}\left(u_{i}^{3}+u_{i} w_{i}^{3}\right)
$$

which corresponds to the exactly solvable model $\left(16^{3} \cdot 4^{2}\right)_{E_{7}^{3} \otimes D^{2}}$ and is of a type different from the ones discussed above. The nine-dimensional noncritical manifold

$$
\mathbb{P}_{(3,2,3,2,3,2,3,3,3,3)}[9]
$$


leads, via its singularity structure, to the five-dimensional critical manifold

$$
\begin{aligned}
& \mathbb{P}_{1} \\
& \mathbb{P}_{2} \\
& \mathbb{P}_{4}
\end{aligned}\left[\begin{array}{lll}
2 & 0 & 0 \\
0 & 3 & 0 \\
1 & 1 & 3
\end{array}\right]
$$

It is crucial that a polynomial was chosen which is not of Brieskorn-Pham type for the last four coordinates in the noncritical manifold.

There are of course other types of weighted $n$-folds which solve the conditions (2.4). An example that does not belong the class above is

$$
\mathbb{P}_{(2,1,2,1,1,1)}[4] \ni\left\{\sum_{i=1}^{2}\left(x_{i}^{2}+x_{i} y_{i}^{2}\right)+x_{5}^{4}+x_{6}^{4}=0\right\}
$$

which leads to the critical surface

$$
\mathbb{P}_{1} \mathbb{P}_{(2,2,1,1)}\left[\begin{array}{ll}
2 & 0 \\
2 & 4
\end{array}\right]
$$

The cohomology of the noncritical 4 -fold is easy to determine: since the $\mathbb{Z}_{2}$-singular set consists of two points, the resolution of which introduces two $(1,1)$-forms. Thus $h^{(1,1)}=3$. With $c_{4}=25 h^{4}$ one finds $\chi_{s}=25$ and hence

$$
\chi=2\left(1+h^{(1,1)}\right)-2=25-1+4=28 .
$$

Thus there appears, in general, not just one additional Kähler mode in the spectrum of the higher dimensional manifold but more new fields whose (possible) interpretation remains obscure at this point.

A final higher dimensional example involving a 4-dimensional critical manifold of a different type starts from the Landau-Ginzburg potential

$$
W=\sum_{i=1}^{3}\left(x_{i}^{3}+x_{i} y_{i}^{3}\right)+\sum_{j=4}^{5} x_{i}^{6}
$$

with central charge $c=12$ which corresponds to the tensor model $16_{E}^{3} 4_{A}^{2}$ and belongs to the configuration

$$
\mathbb{P}_{(6,4,6,4,6,4,3,3)}[18] \text {. }
$$

The 4-dimensional manifold derived from this Landau-Ginzburg configuration belongs to the configuration class

$$
\mathbb{P}_{2} \mathbb{P}_{(2,2,2,1,1)}\left[\begin{array}{ll}
3 & 0 \\
2 & 6
\end{array}\right]
$$


which is indeed a Calabi-Yau deformation class.

This brief discussion shows that many different types of special Fano manifolds feature a structure which makes them amenable to our construction, including a number of infinite series.

\section{Cohomology of Special Fano Spaces and Critical Spectra}

In this Section I will describe in a more complete and coherent way the cohomology of the higher dimensional manifolds and its relation to the critical spectrum. The results described in the previous Sections suggest that the spectrum of the critical vacuum (not described by a CalabiYau manifold in general) is embedded in the cohomology of the higher dimensional space as:

$$
H^{(p, q)}\left(V_{D_{c r i t}}\right) \subset H^{(p+Q-1, q+Q-1)}\left(\mathcal{M}_{D_{\text {crit }}+2(Q-1)}\right)
$$

or, put differently, there exists a projection $\pi$ such that

$$
\pi\left(H^{(p, q)}\left(\mathcal{M}_{D_{c r i t}+2(Q-1)}\right)\right)=H^{(p-(Q-1), q-(Q-1))}\left(V_{D_{c r i t}}\right) .
$$

What has been shown in [18 and elaborated upon in the previous Sections of the present paper is that there exists a geometrical framework for this projection.

There are two main results that follow from our geometrical construction. First:

- The critical spectrum of a $c=D_{\text {crit }}$ vacuum of the $N=1$ spacetime supersymmetric string is determined by the cohomology carried by the singular sets of special Fano varieties of complex dimension $D_{\text {crit }}+2(Q-1)$ together with the monomials of the integrally charged subring of the polynomial (chiral) ring.

This is in contrast to Calabi-Yau manifolds where the spectrum is described by the resolution of the singularities and the polynomial ring. From this an immediate consequence is that

- The cohomology of the higher dimensional ambient weighted projective space does not contribute to the critical spectrum. 
By using a combination of different techniques it is possible to compute the Euler number and, more detailed, the cohomology groups of the higher dimensional spaces. First, one may recall that one way to compute the Euler number of weighted complete intersection Calabi-Yau manifolds is to consider them as coverings of the weighted spaces themselves.

Consider a Fermat configuration

$$
\mathbb{P}_{\left(k_{1}, \ldots, k_{N+2}\right)}[d] \ni\left\{p=\sum_{i} z_{i}^{a_{i}}=0\right\} .
$$

Write this as

$$
z_{1}^{a_{1}}=-z_{2}^{a_{2}}-\cdots-z_{N+2}^{a_{N+2}} .
$$

Clearly this describes an $a_{1}$-fold covering of

$$
\mathbb{P}_{\left(k_{2}, \ldots, k_{N+2}\right)}
$$

as long as $p_{1}$ does not vanish identically, the solution simply being

$$
z_{1}=\left(p_{1}\left(z_{2}, \ldots, z_{N+2}\right)\right)^{1 / a_{1}}, \quad\left(z_{2}, \ldots, z_{N+2}\right) \in \mathbb{P}_{\left(k_{2}, \ldots, k_{N+2}\right)}
$$

When $p_{1}=0$, the only solution is $z_{1}=0$. Thus the space of solutions of the equation $p=0$ becomes a $a_{1}$-fold covering of $\mathbb{P}_{\left(k_{2}, \ldots, k_{N+2}\right)}$ branched over the hypersurface

$$
\mathbb{P}_{\left(k_{2}, \ldots, k_{N+2}\right)}[d]
$$

This is an example of a general situation: if $M$ is an $n$-fold covering of the space $N$ branched over a submanifold $B \in N$ then the Euler number of the spaces involved are related via

Riemann-Hurwitz: $\chi(M)=n \chi(N)-(n-1) \chi(B)$.

This is the result when $M$ is a smooth manifold. In the cases at hand however the manifolds are singular in general and Riemann-Hurwitz computes the Euler number for the singular manifold which has to be resolved: if the manifold has orbifold singularities $S_{i}$ with respect to cyclic groups $\mathbb{Z}_{n_{i}}$ of order $n_{i}$ then the Euler number of the resolved manifold $\tilde{M}$ is given by

$$
\begin{aligned}
\chi(\tilde{M}) & =\chi(M)+\sum_{i}\left(n_{i}-1\right) \chi\left(S_{i}\right) \\
& =n \chi(N)-(n-1) \chi(B)+\sum_{i}\left(n_{i}-1\right) \chi\left(S_{i}\right) .
\end{aligned}
$$


A simple but important example, as will become presently, is the 3 -fold

$$
\mathbb{P}_{(2,2,2,3,3)}[6] \ni\left\{\sum_{i} x_{i}^{3}+\sum y_{j}^{2}=0\right\} .
$$

The singular sets consist of the singular curve

$$
\mathbb{Z}_{2}: \quad \mathbb{P}_{2}[3],
$$

which describes the torus, and two $\mathbb{Z}_{3}$-singular points

$$
\mathbb{Z}_{3}: \quad \mathbb{P}_{1}[2]=2 \text { pts. }
$$

The novel feature of this manifold is the fact that the fiber, which plays such an important rôle in the construction of Section 3, collapses to two points and hence the critical manifold becomes

$$
\begin{aligned}
& \mathbb{P}_{2} \\
& \mathbb{P}_{1}
\end{aligned}\left[\begin{array}{ll}
3 & 0 \\
0 & 2
\end{array}\right]
$$

i.e. it consists of two copies of the torus.

The noncritical spectrum can be obtained as follows: first one computes via Riemann-Hurwitz the Euler number, which turns out to be

$$
\chi_{s}=2 \cdot 4-\chi\left(\mathbb{P}_{2,2,2,3)}[6]\right)=8-2 \cdot 3+\chi\left(\mathbb{P}_{2}[3]\right)=2 .
$$

Resolving the singular space leads to

$$
\chi=-\chi_{s}+\sum_{i}\left(n_{i}-1\right) \chi\left(S_{i}\right)=2+2 \cdot 2=6 .
$$

This result can be checked with the standard resolution formula for the Euler number, using $c_{3}=8 h^{3}$,

$$
\chi=\frac{8 \cdot 6}{8 \cdot 9}-\frac{2}{3}+3 \cdot 2=6 .
$$

Resolving these singularities leads to the surface with the local fibering

$$
\mathbb{P}_{2}[3] \times \mathbb{P}_{1},
$$

which, via Künneth, leads to one additional $(1,1)$-form wheras the resolution of the two $\mathbb{Z}_{3}$ points introduces 2 additional $(1,1)$-forms. Using the result for the Euler number then leads to

$$
\chi=6=2(1+4)-2 h^{(2,1)}
$$


and hence to $h^{(4,1)}=4$.

Thus the Hodge diamond of the 3 -fold is completely determined and leads to (including the 1 monomial of charge 1)

$\begin{array}{ccccccc} & & & 1 & & & \\ & & 0 & & 0 & & \\ 0 & 0 & & 4 & & 0 & \\ & & 2 & & 2 & & 0 \\ & 0 & & 4 & & 0 & \\ & & 0 & & 0 & & \\ & & & 1 & & & \end{array}$

This simple example is interesting because it features a new phenomenon which also occurs for critical vacua in the physical dimension, i.e. for special Fano folds that lead to Calabi-Yau manifolds of dimension $\operatorname{dim}_{\mathbb{C}} M=3$. The important consequence is that the higher dimensional analog of the critical holomorphic $\left(D_{\text {crit }}, 0\right)$ is not always 1-dimensional, a fact for which our construction provides a simple geometrical explanation, the reason being that multiple copies of the critical manifold can be embedded in the higher dimensional space.

An example that leads to a phenomenological interesting string vacuum is furnished by

$$
\mathbb{P}_{(2,2,2,3,3,3,3)}[9] \ni\left\{\sum_{i}\left(x_{i}^{3} y_{i}+y_{i}^{3}\right)+x_{4}^{3}=0\right\} .
$$

There are two singular surfaces embedded in this manifold, the projective plane and the cubic surface

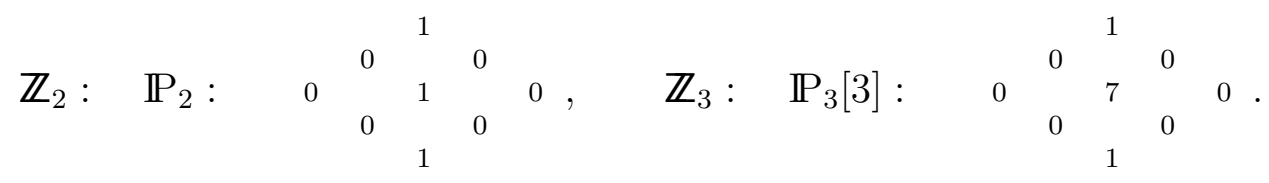

When resolved, these lead to the 3 -folds

$$
\mathbb{P}_{2} \times \mathbb{P}_{1}, \quad \mathbb{P}_{3}[3] \times \mathbb{P}_{1}
$$

respectively. Using Künneth's formula these 3 -folds lead to the following Hodge diamond of the resolved higher dimensional Fano manifold of special type

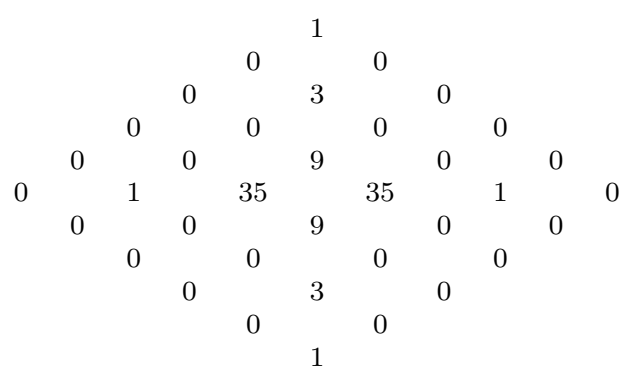


Thus the Euler number becomes

$$
\chi=2\left(b_{0}-b_{1}+b_{2}-b_{3}+b_{4}\right)-b_{5}=-46 .
$$

The critical manifold

$$
\begin{aligned}
& \mathbb{P}_{2} \\
& \mathbb{P}_{3}
\end{aligned}\left[\begin{array}{ll}
3 & 0 \\
1 & 3
\end{array}\right]
$$

which is embedded in the special Fano 5-fold is of some interest because it is the starting point of the construction of a 3-generation Calabi-Yau manifold introduced in [46].

This example is misleading in one respect however: all of the critical generations are parametrized by the chiral ring itself, i.e. the monomials of charge 1. This translates into the fact that all complex deformations of the critical Calabi-Yau space (5.22) which derives from this Fano fold via our construction are indeed parametrized by the monomials of the polynomials. In many cases however these monomials fail to parametrize all complex deformations, a simple example being furnished by the complete intersection Calabi-Yau manifold [50]

$$
\mathbb{P}_{1}\left[\begin{array}{ll}
2 & 0 \\
\mathbb{P}_{4} & 4
\end{array}\right]
$$

This critical manifold can be derived from the higher dimensional space

$$
\mathbb{P}_{(2,2,2,2,2,3,3)}[8] \ni\left\{\sum_{i=1}^{2}\left(x_{i}^{4}+x_{i} y_{i}^{2}\right)+x_{3}^{4}+x_{4}^{4}+x_{5}^{4}=0\right\},
$$

the analysis of which shows that indeed the 'missing' complex deformations, not parametrized by the complex monomials, originate from the singularities of the higher dimensional space.

It should be realized that the computation of the critical spectrum from the higher dimensional space does not depend on the ability to find an embedding of a 3-dimensional Calabi-Yau space. Consider, e.g.

$$
\mathbb{P}_{(1,1,2,2,2,2,2)}[6] \ni\left\{z_{1}^{6}+z_{2}^{6}+\sum_{i} z_{i}^{3}=0\right\}
$$

for which the Riemann-Hurwitz formula leads to the singular Euler number

$$
\chi_{s}=3 \cdot 6-2 \chi\left(\mathbb{P}_{1,1,2,2,2,2)}[6]\right)=18-2\left\{3 \cdot 5-2 \chi\left(\mathbb{P}_{(1,1,2,2,2)}[6]\right)\right\}=\cdots=-132
$$

which in turn leads to the resolved Euler number

$$
\chi=-132+\chi\left(\mathbb{P}_{4}[3]\right)=-138
$$


after blowing up the singular cubic 3 -fold.

From the singular cubic 3-fold, which has Euler number -6 one obtains immediately that the number of critical $(1,1)$-forms is 1 and that there are 5 additional critical $(2,1)$-modes coming from the singularity, in addition to the 68 monomial deformations. Thus for the total critical spectrum one obtains

$$
\left(h_{\text {crit }}^{(1,1)}, h_{\text {crit }}^{(2,1)}\right)\left(\mathbb{P}_{(1,1,2,2,2,2,2)}[6]\right)=(1,73)
$$

which is the correct string spectrum.

It is also of interest to compute the cohomology of the noncritical manifold. In order to obtain the cohomology groups of the ambient space it is only necessary to compute, via Künneth the Hodge diamond of the bundle $\mathbb{P}_{3}[4] \times \mathbb{P}_{1}$, leading to one additional $(1,1)$-form. Using the result for the Euler number one finds

$$
-138=\chi=2\left(b_{0}+b_{2}+b_{4}\right)-b_{5}=10-2 h^{(4,1)}-146
$$

and hence $h^{(4,1)}=1$, as expected. Thus the Hodge diamond

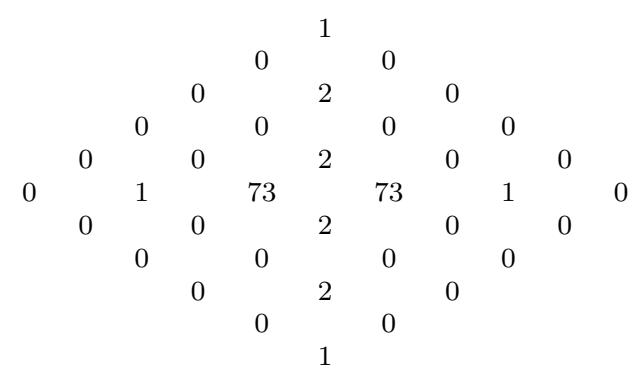

is obtained.

The general Hodge-diamond for odd-dimensional manifolds, $\operatorname{dim}_{\mathbb{C}} M=(2 p+1)$, is

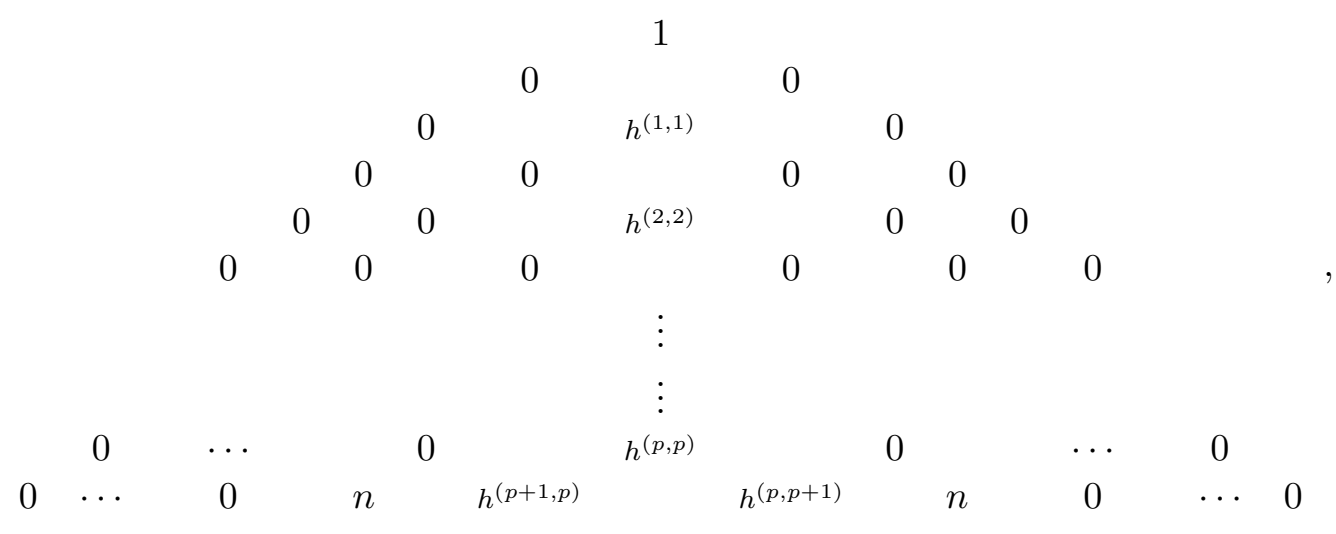


whereas for even-dimensional manifolds, $\operatorname{dim}_{\mathbb{C}}=2 p$, it is of the form

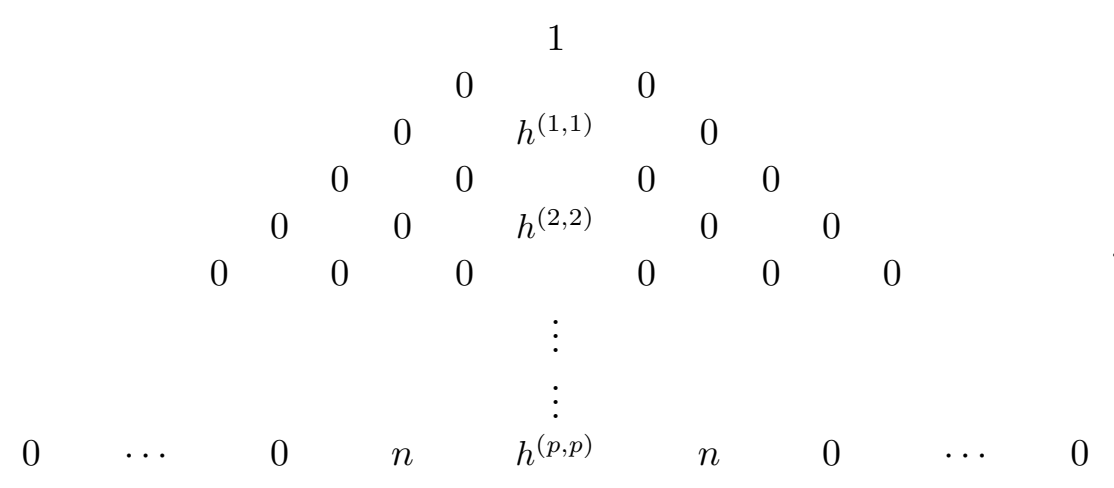

Even though, from an abstract cohomological point of view, it is not known how to perform the projection of the special Fano Hodge diamond to the critical stringy part, our construction provides a geometrical prescription which does provide a universal tool to perform the decomposition of the ambient cohomology into a stringy and a remaining part. One universal feature which follows from this geometrical construction is the fact that in the transition from the higher dimensional spectrum to the critical spectrum, described by the generations and antigenerations, the cohomology of the ambient projective space does not contribute.

\section{When $h^{\left.D_{\text {crit }}+Q-1, Q-1\right)}>1$ : a Degeneration Phenomenon.}

It is tempting to try a cohomological definition of the special class of Fano folds (2.4) as a class of spaces for which there exists precisely one analog of the holomorphic $\left(D_{\text {crit }}, 0\right)$-form which exists in critical string vacua. This however would be fallacious.

Consider the real life example

$$
\mathbb{P}_{(2,2,2,2,2,5,5)}[6] \ni\left\{\sum_{i} x_{i}^{3}+\sum y_{j}^{2}=0\right\}
$$

whose singular sets consist of the 3 -fold

$$
\mathbb{Z}_{2}: \quad \mathbb{P}_{4}[5], \quad \chi=-200
$$

and the two points

$$
\mathbb{Z}_{5}: \quad \mathbb{P}_{1}[2]=2 \text { pts. }
$$


Going back to the discussion of Sections 3 and 4, we see that the fiber collapses down to two points in this example and the critical manifold becomes

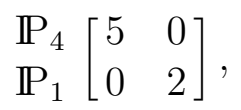

i.e. it consists of two copies of the quintic.

This degeneration phenomenon also shows up through an analysis of the Hodge structure of the manifold. The noncritical spectrum can be obtained as follows: first one computes via Riemann-Hurwitz the Euler number, which turns out to be

$$
\chi_{s}=2 \cdot 6-\chi\left(\mathbb{P}_{2,2,2,2,2,3)}[10]\right)=-198
$$

which, when resolved, leads to

$$
\chi=-\chi_{s}+\sum_{i}\left(n_{i}-1\right) \chi\left(S_{i}\right)=-198+(-200)+4 \cdot 2=-390
$$

This result can be checked with the resolution formula for the Euler number: with $c_{5}=-7968 h^{5}$ it follows that

$$
\chi=-\frac{498}{5}-\frac{-200}{2}+2(-200)-\frac{2}{5}+5 \cdot 2=-390 .
$$

In more detail, by resolving these singularities, one is locally lead to the 4 -fold

$$
\mathbb{P}_{4}[5] \times \mathbb{P}_{1}
$$

which, via Künneth, leads to one additional $(1,1)$-form whereas the resolution of the two $\mathbb{Z}_{5}$ points introduces 2 additional $(1,1)$-forms. Using the above result for the Euler number then leads to

$$
\chi=-390=2(1+4+4)-2 h^{(4,1)}-404
$$

and hence to $h^{(4,1)}=2$.

Thus the Hodge diamond of the 5 -folds is completely determined and leads to

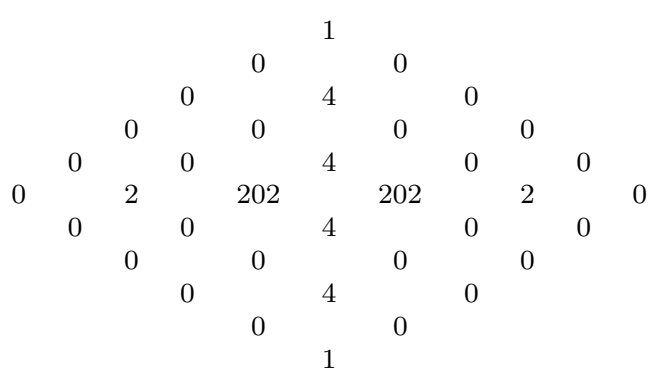


(including the 101 monomials of charge unity).

This example is important because it shows two things: the first is that also for the physically relevant situation of Calabi-Yau manifolds of three complex dimensions it can happen that $h^{\left(D_{\text {crit }}+Q-1, Q-1\right)}>1$ ! This shows that, contrary to what one might expect, the higher dimensional analog of the critical holomorphic $\left(D_{\text {crit }}, 0\right)$ is not always 1-dimensional, the simple reason being that multiple copies of the critical manifold can be embedded in the higher dimensional space. The second point is that, contrary to the case of smooth noncritical manifolds such as $\mathbb{P}_{8}[3]$ and $\mathbb{P}_{(1,1,1,1,1,1,2)}[4]$, singular spaces have a much more complicated cohomology. Hence such manifolds lead, in general, to many more states than those comprising the critical spectrum.

\section{Splitting and Contraction: Many Fano Fold Embedding of Critical Vacua}

In this Section it is pointed out that the relation between higher dimensional manifolds and critical vacua is not $1-1$. Instead many higher dimensional manifolds are related to one and the same critical vacuum. In general even an infinite number.

One way to see this, is via the process of splitting and contraction introduced in [43]. The discussion of ref. [43] is restricted to Calabi-Yau manifolds embedded in products of ordinary projective spaces but it readily generalizes to the more general framework of weighted projective spaces.

It is useful to start with an example. Consider the 7 -fold

$$
\mathbb{P}_{(1,1,10,10,2,2,2,2,6)}[12] \ni\left\{\sum_{i=1}^{2}\left(x_{i}^{2} y_{i}+y_{i} z_{i}+z_{i}^{6}\right)+z_{3}^{6}+z_{4}^{6}+z_{5}^{2}=0\right\}
$$

where $\left(x_{1}, x_{2}, y_{1}, y_{2}, z_{1}, z_{2}, \ldots, z_{5}\right)$ are the coordinates of the ambient weighted space. This space leads, via an iterative construction, as described in the previous Section, to the critical manifold

$$
\begin{aligned}
& \mathbb{P}_{(1,1)} \\
& \mathbb{P}_{(1,1)} \\
& \mathbb{P}_{(1,1,1,1,3)}
\end{aligned} \quad\left[\begin{array}{lll}
2 & 0 & 0 \\
1 & 1 & 0 \\
0 & 1 & 6
\end{array}\right]
$$

with the by now familiar split of the polynomial. 
Using the same analysis as described in 43 it is however easy to see that this critical manifold is in fact isomorphic to the Calabi-Yau manifold

$$
\mathbb{P}_{(1,1)} \mathbb{P}_{(1,1,1,1,3)}\left[\begin{array}{ll}
2 & 0 \\
1 & 6
\end{array}\right]
$$

described by the polynomials

$$
\begin{aligned}
& p_{1}=x_{1}^{2} y_{1}+x_{2}^{2} y_{2}, \\
& p_{2}=y_{1}^{6}+y_{2}^{6}+y_{3}^{6}+y_{4}^{6}+y_{5}^{2}
\end{aligned}
$$

which can be derived via our construction from the higher dimensional manifold

$$
\mathbb{P}_{(5,5,2,2,2,2,6)}[12] \ni\left\{\sum_{i=1}^{2}\left(x_{i}^{2} y_{i}+y_{i}^{6}\right)+y_{3}^{6}+y_{4}^{6}+y_{5}^{2}=0\right\}
$$

where $\left(x_{1}, x_{2}, y_{1}, y_{2}, \ldots, y_{5}\right)$ are the homogeneous coordinates of the 6 -dimensional weighted space. Manifold (7.2), in fact, is the so-called split [43] of the manifold (7.3) and in special circumstances, which apply in the present case, the splitting or contraction process does not change the theory but rather provides different representations of the same manifold. In such a situation one is dealing with a split that is said to be ineffective.

It turns out that the manifold (7.3) can be represented via ineffective splits in an infinite number of ways as

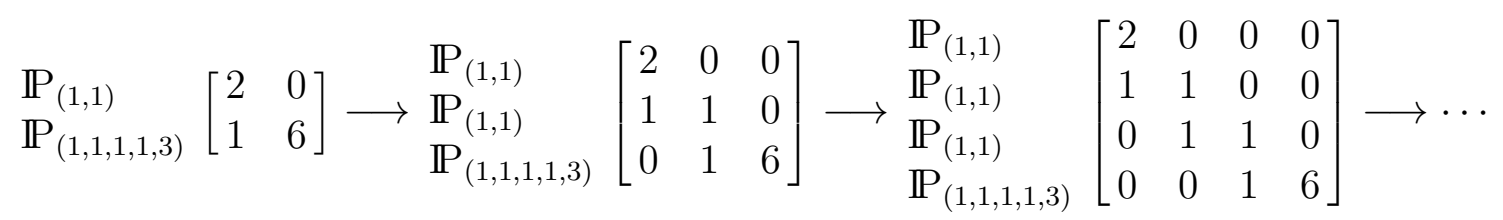

This chain of different representations of the same Calabi-Yau 3-fold can be derived from a corresponding sequence of higher dimensional special Fano spaces

$$
\mathbb{P}_{(5,5,2,2,2,2,6)}[12] \longrightarrow \mathbb{P}_{(1,1,10,10,2,2,2,2,6)}[12] \longrightarrow \mathbb{P}_{(5,5,2,2,10,10,2,2,2,2,6)}[12] \longrightarrow \cdots
$$

i.e. the infinite sequence of

$$
\mathbb{P}_{(5,5,2,2,10,10,2,2,10,10, \ldots, 2,2,10,10,2,2,6)}[12]
$$

where the part $(2,2,10,10)$ occurs $k$ times, and

$$
\mathbb{P}_{(1,1,10,10,2,2,10,10,2,2, \ldots 10,10,2,2,2,2,6)}[12]
$$


where $(10,10,2,2)$ occurs $k$ times. The burden of this observation is that a given string vacuum can be derived from an infinite sequence of special Fano manifolds with ever increasing dimension.

This example easily generalizes to a class of spaces [47] which was mentioned already in Section 3. Consider manifolds embedded in

$$
\begin{aligned}
& \mathbb{P}_{(1,1)} \\
& \mathbb{P}_{\left(k_{1}, k_{1}, k_{3}, k_{4}, k_{5}\right)}
\end{aligned}\left[\begin{array}{cc}
2 & 0 \\
k_{1} & k
\end{array}\right]
$$

where $k=k_{1}+k_{3}+k_{4}+k_{5}$. These spaces can be split into the infinite sequences

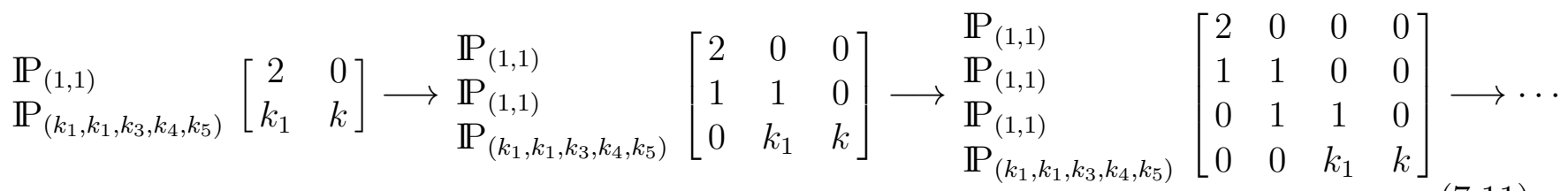

with the corresponding sequence of higher dimensional spaces given by

$$
\mathbb{P}_{\left(k-k_{1}, k-k_{1}, 2 k_{1}, 2 k_{1}, 2 k_{3}, 2 k_{4}, 2 k_{5}\right)}[2 k] \longrightarrow \mathbb{P}_{\left(k_{1}, k_{1}, 2\left(k-k_{1}\right), 2\left(k-k_{1}\right), 2 k_{1}, 2 k_{1}, 2 k_{3}, 2 k_{4}, 2 k_{5}\right)}[2 k] \longrightarrow \cdots
$$

i.e. they belong to the set of spaces of type

$$
\mathbb{P}_{\left(\left(k-k_{1}\right),\left(k-k_{1}\right), 2 k_{1}, 2 k_{1}, 2\left(k-k_{1}\right), 2\left(k-k_{1}\right), \ldots ., 2 k_{1}, 2 k_{1}, 2 k_{3}, 2 k_{4}, 2 k_{5}\right)}[2 k]
$$

where the part $\left(2 k_{1}, 2 k_{1}, 2\left(k-k_{1}\right), 2\left(k-k_{1}\right)\right)$ occurs $p$ times, and

$$
\mathbb{P}_{\left(k_{1}, k_{1}, 2\left(k-k_{1}\right), 2\left(k-k_{1}\right), 2 k_{1}, 2 k_{1}, \ldots, 2 k_{3}, 2 k_{4}, 2 k_{5}\right)}[2 k],
$$

where $\left(2\left(k-k_{1}\right), 2\left(k-k_{1}\right), 2 k_{1}, 2 k_{1}\right)$ occurs $p$ times.

\section{Topological Relations between Fano Varieties}

It is the purpose of this short Section to point out that certain topological identities, such as those employed in 43], make it possible to apply our embedding construction of Section 3 to Calabi-Yau manifolds for which naively an analysis along the present lines appears impossible. The reason for this is that these topological identities allow us to represent complete intersection Calabi-Yau manifolds in many different ways. 
Consider the codimension-four Calabi-Yau manifold

$\mathbb{P}_{1}$
$\mathbb{P}_{2}$
$\mathbb{P}_{2}$
$\mathbb{P}_{2}$$\left[\begin{array}{llll}2 & 0 & 0 & 0 \\ 1 & 2 & 0 & 0 \\ 0 & 1 & 2 & 0 \\ 0 & 0 & 1 & 2\end{array}\right]$

with the defining polynomials

$$
\begin{aligned}
& p_{1}=\sum_{i=1}^{2} u_{i}^{2} v_{i}, \quad p_{2}=\sum_{i=1}^{3} v_{i}^{2} w_{i} \\
& p_{3}=\sum_{i=1}^{3} w_{i}^{2} x_{i}, \quad p_{4}=\sum_{i=1}^{3} x_{i}^{2}
\end{aligned}
$$

which can be derived from

$$
\mathbb{P}_{(5,5,6,6,6,4,4,4,8,8,8)}[16]
$$

as discussed in Section 4.

This manifold can be represented in rather different ways by using a well-known representation of the sphere as a quadric in the projective plane

$$
S^{2}=\mathbb{P}_{1}=\mathbb{P}_{2}[2]
$$

Using this, the manifold (8.1) can be rewritten as

$$
\begin{aligned}
& \mathbb{P}_{1} \\
& \mathbb{P}_{2} \\
& \mathbb{P}_{2} \\
& \mathbb{P}_{1}
\end{aligned}\left[\begin{array}{lll}
2 & 0 & 0 \\
1 & 2 & 0 \\
0 & 1 & 2 \\
0 & 0 & 2
\end{array}\right]
$$

Furthermore one can use the surface identity 43]

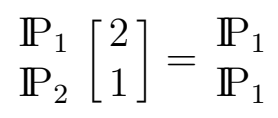

via the application rule

$$
\begin{gathered}
\mathbb{P}_{1} \\
\mathbb{P}_{2} \\
X
\end{gathered}\left[\begin{array}{cc}
2 & 0 \\
1 & a \\
0 & M
\end{array}\right]=\begin{gathered}
\mathbb{P}_{1} \\
\mathbb{P}_{1} \\
X
\end{gathered}\left[\begin{array}{c}
a \\
a \\
M
\end{array}\right]
$$

to obtain the representation

$$
\begin{aligned}
& \mathbb{P}_{1} \\
& \mathbb{P}_{1} \\
& \mathbb{P}_{1} \\
& \mathbb{P}_{2}
\end{aligned}\left[\begin{array}{ll}
2 & 0 \\
2 & 0 \\
0 & 2 \\
1 & 2
\end{array}\right]
$$

whose defining polynomials are some deformations of

$$
\begin{aligned}
& p_{1}=\sum_{i} u_{i}^{2} v_{i}^{2} y_{i} \\
& p_{2}=\sum_{i} x_{i}^{2} y_{i}^{2} .
\end{aligned}
$$


The natural ansatz for the higher dimensional polynomial

$$
p=p_{1}+p_{2}
$$

clearly does not have an isolated singularity and hence does not have an interpretation as the defining equation of a quasihomogeneous hypersurface in some weighted projective space. In fact it is not even possible to assign weights to the variables such that a weighted projective space could be specified in which the purported hypersurface is supposed to live. But because this manifold is diffeomorphic to the variety (8.1) it does admit, via this equivalence, an embedding into a higher dimensional space.

\section{Landau-Ginzburg Theories, Special Fano Manifolds, and Critical Vacua}

The basic idea in [18] is that it is the singularity structure of the higher dimensional special Fano manifolds (and the polynomial ring) that determines the string physics. This idea also leads to a new way of looking at the Calabi-Yau/Landau-Ginzburg connection as will be discussed presently.

The starting point of the mean field description of $\mathrm{N}=2$ superconformal theory is an $\mathrm{N}=2$ supersymmetric field theory in terms of a set of chiral superfields $\Phi_{i}\left(z, \bar{z}, \theta^{ \pm}, \bar{\theta}^{ \pm}\right)$, defined on the superspace parametrized by coordinates $\left(z, \bar{z}, \theta^{ \pm}, \bar{\theta}^{ \pm}\right)$via the constraints

$$
D^{+} \Phi_{i}=0=\bar{D}^{+} \Phi_{i}
$$

where

$$
D^{ \pm}=\frac{\partial}{\partial \theta^{ \pm}}+\theta^{\mp} \partial_{z}
$$

(and similarly for the right moving sector).

The action for these fields will be chosen to be of the form

$$
\int d^{2} z d^{2} \theta d^{2} \bar{\theta} K\left(\Phi_{i}, \bar{\Phi}_{i}\right)+\int d^{2} z d^{2} \theta W\left(\Phi_{i}\right)+\text { c.c. }
$$

where $K$ is the Kähler potential and $W$ is the superpotential which is assumed to be quasihomogeneous. This means that there exists a set of weights $k_{i}>0$ such that under $\Phi_{i} \rightarrow \lambda^{k_{i}} \Phi_{i}$ the superpotential scales as

$$
W\left(\lambda^{k_{i}} \Phi_{i}\right)=\lambda^{d} W\left(\Phi_{i}\right)
$$


for some integer $d$.

It was the insight of Martinec [8] and Vafa and Warner [9] that such Landau-Ginzburg theories are useful for the understanding of string vacua and that much information about such groundstates is already encoded in the superpotential. The point that was emphasized by these authors is that since the Kähler potential contains only irrelevant and marginal operators the superpotential $W$ already determines essential properties of the theory.

The important structure determined by the superpotential is the ring of monomials that one can build with the order parameters $\Phi_{i}$. This ring is defined by all monomials modulo those that are generated by the ideal $\mathcal{I}$ defined by the partial derivatives $\partial_{i} W$ of the superpotential with respect to the order fields $\Phi_{i}$

$$
\mathcal{R}=\frac{\mathbb{C}\left[\Phi_{i}\right]}{\mathcal{I}\left[\partial_{i} W\right]}
$$

Since this ring is finite dimensional, its dimension given by the Milnor number

$$
\mu=\prod_{i}\left(\frac{k_{i}}{d}-1\right),
$$

there must exist a monomial of maximal degree. This monomial is important because it corresponds to the field of maximal weight $\Delta=c / 3$ that always exists in an $N=2$ supersymmetric conformal field theory. Using the fact of Arnold that the maximal degree obtainable is determined by

$$
d_{\text {max }}=\sum_{i=1}^{N}\left(1-2 q_{i}\right),
$$

it follows 48 that the central charge of such a Landau-Ginzburg theory is

$$
c=3 \sum_{i=1}^{N}\left(1-2 q_{i}\right),
$$

where $q_{i}=k_{i} / d$.

The concept of Landau-Ginzburg theories of course allows to describe compactifications of the heterotic string not only from ten dimensions down to four dimensions by using a Landau-Ginzburg theory of central charge nine, but also allows to describe compactifications down to six dimensons via theories with central charge six, or to eight spacetime dimensions with theories of central charge three. The critical dimension of the internal theory, if it has a spacetime interpretation, in all these case is

$$
D_{\text {crit }}=c / 3 \text {. }
$$


In the context of Landau-Ginzburg theories the integer $Q$ introduced in Section 2 is simply the total charge and one can rewrite the central charge as

$$
N=D_{\text {crit }}+2 Q
$$

thus determining the number of scaling fields in terms of the critical dimension and the total charge.

If $D_{\text {crit }}$ is an integer then clearly $2 Q \in \mathbb{N}$ and it is always possible to add an additional trivial field in order to obtain integer total charge $Q \in \mathbb{N}$. As mentioned already $Q$ has the geometrical interpretation of a codimension: it describes into how many polynomial constraints the superpotential must split in order to define a manifold of critical dimension $D_{\text {crit }}$. Given the number of constraints, the critical dimension, and the number of fields in the theory, it follows that the number of projective scales is

$$
\mathcal{F}=N-D_{\text {crit }}-Q
$$

and therefore

$$
\mathcal{F}=Q
$$

Consider now the path integral

$$
Z=\int \prod_{i} D \Phi_{i} \prod_{j} D \Psi_{j} e^{-\int d^{2} z d^{4} \theta K\left(\Phi_{i}, \bar{\Phi}_{i}, \Psi_{j}, \bar{\Psi}_{j}\right)+\int d^{2} z d^{2} \theta W\left(\Phi_{i}, \Psi_{i}\right)+c . c .}
$$

with the superpotential

$$
W\left(\Phi_{i}, \Psi_{i}\right)=\sum_{i=1}^{3} \Psi_{i}^{3} \Phi_{i}+\sum_{j=1}^{4} \Phi_{j}^{3} .
$$

A priori there is no reason to ask for a geometrical description of the conformal fixed points of this model. However this theory in itself does not describe a string ground state, the missing ingredient being the GSO projection. Implementation of this projection in this context amounts to compactifying the affine variety and hence the question arises what spacetime interpretation the resulting theory affords.

This is were hindsight comes in. By computing the spectrum of the GSO projected LandauGinzburg theory [49] and comparing the result with known Calabi-Yau manifolds it becomes obvious that the theory (9.14) should related to a manifold embedded in the configuration 46.

$$
\begin{aligned}
& \mathbb{P}_{2} \\
& \mathbb{P}_{3}
\end{aligned}\left[\begin{array}{ll}
3 & 0 \\
1 & 3
\end{array}\right]
$$


and defined by the polynomial constraints

$$
\begin{aligned}
& p_{1}=\sum_{i=1}^{3} y_{i} z_{i}^{3}, \\
& p_{2}=\sum_{j=1}^{4} y_{i}^{3}
\end{aligned}
$$

where the superpotential $W$ has been split into two parts. This is supported by an analysis of the underlying exactly solvable model of the potential (9.14). Indeed, it was shown by Gepner [6] [0], even before the appearance of [8] [9], through a detailed analysis of the spectrum and the symmetries that the manifold (9.15) is related to the GSO-projected tensor model

$$
\left(1 \cdot 16^{3}\right)_{A_{2} \otimes E_{7}^{3}}
$$

of $\mathrm{N}=2$ exactly solvable minimal models at level 1 and level 16 , endowed with the diagonal modular invariant and the $E_{7}$ invariant respectively. The results of [8] [9] support this connection.

Knowing what to shoot for then helps in massaging the path integral above into a form which suggests a relation to (9.15). The idea 450 is to obtain some $\delta$-function constraints, restricting the variables to lie on a hypersurface. To achieve this, define new coordinates

$$
\xi_{1}=\Phi_{1}^{3}, \quad \xi_{i}=\frac{\Phi_{i}}{\Phi_{1}}, i=2, \ldots, 4, \xi_{5}=\Phi_{1} \Psi_{1}^{3}, \quad \xi_{6}=\frac{\Psi_{2}}{\Psi_{1}}, \quad \xi_{7}=\frac{\Psi_{3}}{\Psi_{1}} .
$$

As emphasized in [45] it is important for this coordinate change to lead to a constant Jacobian. Because of this we can write (neglecting the kinetic term)

$$
\begin{aligned}
Z & =\int \prod_{i} D \Phi_{i} \prod_{j} D \Psi_{j} e^{-\int d^{2} z d^{2} \theta W\left(\Phi_{i}, \Psi_{j}\right)+c . c .} \\
& \sim \int \prod D \xi_{i} e^{-\int d^{2} z d^{2} \theta W\left(\xi_{i}\right)+c . c .}
\end{aligned}
$$

with superpotential

$$
W=\xi_{5}\left(1+\xi_{1} \xi_{6}^{3}+\xi_{2} \xi_{7}^{3}\right)+\xi_{1}\left(1+\xi_{2}^{3}+\xi_{3}^{3}+\xi_{4}^{3}\right) .
$$

Integrating out $\xi_{1}, \xi_{5}$ then leads to a product of two delta functions whose arguments are interpreted as the two polynomials $p_{1}, p_{2}$ in inhomogeneous coordinates.

A similar analysis can be performed for the manifolds (3.11) of ref. [47]: starting from

$$
W\left(\Phi_{i}, \Psi_{i}\right)=\Phi_{1} \Psi_{1}^{2}+\Phi_{2} \Psi_{2}^{2}+\sum_{i=1}^{5} \Phi_{i}^{l / l_{i}},
$$


one can assume that $\Phi_{1} \neq 0$ and define the new coordinates

$$
\xi_{1}=\Phi_{1}^{l_{1}}, \quad \xi_{i}=\frac{\Phi_{i}}{\Phi_{1}^{l_{i} / l_{1}}}, i=2, \cdots, 5, \quad \xi_{6}=\Phi_{1} \Psi_{1}^{2}, \quad \xi_{7}=\frac{\Psi_{2}}{\Psi_{1}} .
$$

This can be rewritten as

$$
W\left(\xi_{i}\right)=\xi_{6}\left(1+\xi_{2} \xi_{7}^{2}\right)+\xi_{1}\left(1+\sum_{i=2}^{5} \xi_{i}^{l / l_{i}}\right)
$$

and because the Jacobian is constant the path integral has not changed. By integrating out the coordinates $\xi_{1}, \xi_{6}$ one finally obtains the polynomial constraints (written in homogeneous coordinates)

$$
\begin{aligned}
& p_{1}=y_{1} z_{1}^{2}+y_{2} z_{2}^{2}, \\
& p_{2}=\sum_{i=1}^{5} y_{i}^{l / l_{i}} .
\end{aligned}
$$

Interpreting the coordinates $z_{i}$ as the variables of the projective curve and the coordinates $y_{i}$ as the variables of a projective 4 -fold these constraints define a complete intersection in

$$
\mathbb{P}_{\left(l_{1}, l_{2}, l_{3}, l_{4}, l_{5}\right)}\left[\begin{array}{cc}
l_{1} & l \\
2 & 0
\end{array}\right]
$$

where $l=l_{2}+l_{3}+l_{4}+l_{5}$.

This is not the whole story however, since we have not yet computed the spectrum of the theory. By correcting the Euler numbers of these configurations

$$
\chi_{s}=-\frac{2 l}{l_{1} \cdots l_{5}}\left[l_{1}^{3}+\sum_{i=2}^{5} l_{i}^{2} \hat{l}_{i}+\sum_{\substack{i<j<k \\ i, j, k \in\{2, \ldots, 5\}}} 2 l_{i} l_{j} l_{k}\right]
$$

where $\hat{l}_{i}=l-l_{i}$, with the contributions coming from the blow-up of the singular sets one finds agreement with the Landau-Ginzburg result as described in [47]. It is furthermore possible to find the exactly solvable models for these manifolds as well: to each of these manifolds one can associate an exactly solvable model by starting from the weights $l_{i}$ of the weighted projective space. Defining the levels as

$$
k_{1}=k_{2}=\frac{2 l}{l_{1}}-2, \quad k_{i}=\frac{l}{l_{i}}-2, \quad i=3,4,5
$$

one finds for the central charge of these models

$$
c=2 \frac{3 k_{1}}{k_{1}+2}+\sum_{i=3}^{5} \frac{3 k_{i}}{k_{i}+2}=9
$$


as is necessary for an allowed configuration. Finally one may check the spectra of the various incarnations of the individual models.

It is clear from the discussion in Section 3 that via the analysis of the singular sets of the variety, a prescription emerges for the decomposition of the superpotential $W$ into the $Q$ polynomials which eventually define the Calabi-Yau manifold of critical dimension. We may consider the singular sets either in the higher dimensional weighted hypersurface or in the corresponding affine variety, thereby translating the analysis appropriately. For the manifold (9.15) we are then lead to the affine hypersurface

$$
\mathbb{C}_{(2,2,2,3,3,3,3)}[9]
$$

whose singular sets are

$$
\mathbb{C}_{3}, \quad \mathbb{C}_{4}[3]
$$

whereas for the manifolds of type (3.11) we find

$$
\mathbb{C}_{\left(2 k, K-k, 2 k, K-k, 2 k_{3}, 2 k_{4}, 2 k_{5}\right)}[2 K]
$$

with (generic) singular sets

$$
\mathbb{C}_{1}, \quad \mathbb{C}_{\left(k, k, k_{3}, k_{4}, k_{5}\right)}[K] .
$$

The details of the construction of [45] remain in place, of course, but what emerges is the explanation that the path integral in fact localizes on the singular sets of the affine space over which the superpotential lives.

\section{Mirrors of Rigid String Vacua}

In the framework of the Landau-Ginzburg theoretic description of string vacua a number of models appear whose spectrum makes them candidates for mirrors of rigid ground states. Indeed, by applying the construction of [2] it is easy to find the corresponding pairs of Landau-Ginzburg potentials which define the appropriate mirror partners of rigid theories, as will be seen in this Section.

In the list of Landau-Ginzburg theories with isolated singularities [12, 13] there appear two distinct models without antigenerations, both of which were mentioned in Section 3

$$
\left(1^{9}\right)_{A^{9}}^{(0,84)} \leftrightarrow \mathbb{C}_{(1,1,1,1,1,1,1,1,1)}^{*}[3] \ni\left\{W=\sum_{i=1}^{3} z_{i}^{3}=0\right\}
$$


and

$$
\left(2^{6}\right)_{A^{6}}^{(0,90)} \leftrightarrow \mathbb{C}_{(1,1,1,1,1,1,2)}^{*}[4] \ni\left\{W=\sum_{i} z_{i}^{4}+z_{7}^{2}=0\right\}
$$

The mirrors of these theories can be constructed by using the results of [2]. Consider e.g. the orbifold

$$
\mathbb{C}_{(1,1,1,1,1,1,1,1,1)}[3] / \mathbb{Z}_{3}^{6}:\left[\begin{array}{lllllllll}
2 & 1 & 0 & 0 & 0 & 0 & 0 & 0 & 0 \\
0 & 2 & 1 & 0 & 0 & 0 & 0 & 0 & 0 \\
0 & 0 & 2 & 1 & 0 & 0 & 0 & 0 & 0 \\
0 & 0 & 0 & 2 & 1 & 0 & 0 & 0 & 0 \\
0 & 0 & 0 & 0 & 2 & 1 & 0 & 0 & 0 \\
0 & 0 & 0 & 0 & 0 & 2 & 1 & 0 & 0
\end{array}\right] .
$$

of the model $\mathbb{C}_{(1,1,1,1,1,1,1,1,1)}[3]$. This orbifold has the spectrum $\left(h^{(1,1)}, h^{(2,1)}, \chi\right)=(84,0,168)$ and leads, via fractional transformations [2] of the coordinates, to the Landau-Ginzburg potential

$$
\mathbb{C}_{(96,48,72,60,66,63,43,64,64)}[192] \ni\left\{z_{1}^{2}+z_{1} z_{2}^{2}+z_{2} z_{3}^{2}+z_{3} z_{4}^{2}+z_{4} z_{5}^{2}+z_{5} z_{6}^{2}+z_{6} z_{7}^{3}+z_{8}^{3}+z_{9}^{3}=0\right\}
$$

which has the correct spectrum $(84,0,168)$.

Even though the above mirror is certainly a valid representation it is not a unique one. Consider the orbifold

$$
\mathbb{C}_{(1,1,1,1,1,1,1,1,1)}[3] / \mathbb{Z}_{3}^{7}:\left[\begin{array}{ccccccccc}
2 & 1 & 0 & 0 & 0 & 0 & 0 & 0 & 0 \\
0 & 2 & 1 & 0 & 0 & 0 & 0 & 0 & 0 \\
0 & 0 & 2 & 1 & 0 & 0 & 0 & 0 & 0 \\
0 & 0 & 0 & 2 & 1 & 0 & 0 & 0 & 0 \\
0 & 0 & 0 & 0 & 2 & 1 & 0 & 0 & 0 \\
0 & 0 & 0 & 0 & 0 & 2 & 1 & 0 & 0 \\
0 & 0 & 0 & 0 & 0 & 0 & 2 & 1 & 0
\end{array}\right]
$$

which also has the spectrum $(84,0,168)$. Applying fractional transformations again shows that this orbifold is isomorphic to the hypersurface

$\mathbb{C}_{(192,96,144,120,132,126,129,85,128)}[384] \ni\left\{z_{1}^{2}+z_{1} z_{2}^{2}+z_{2} z_{3}^{2}+z_{3} z_{4}^{2}+z_{4} z_{5}^{2}+z_{5} z_{6}^{2}+z_{6} z_{7}^{2}+z_{7} z_{8}^{3}+z_{9}^{3}=0\right\}$.

For the second theory the orbifolding

$$
\mathbb{C}_{(1,1,1,1,1,1,2)}^{*}[4] / \mathbb{Z}_{4}^{3}:\left[\begin{array}{ccccccc}
3 & 1 & 0 & 0 & 0 & 0 & 0 \\
0 & 3 & 1 & 0 & 0 & 0 & 0 \\
0 & 0 & 3 & 1 & 0 & 0 & 0 \\
0 & 0 & 0 & 3 & 1 & 0 & 0
\end{array}\right]
$$

leads to the mirror spectrum and fractional transformations of the variables lead to the mirror potential

$$
\mathbb{C}_{(108,72,84,80,61,81,162)}[324] \ni\left\{z_{1}^{3}+z_{1} z_{2}^{3}+z_{2} z_{3}^{3}+z_{3} z_{4}^{3}+z_{4} z_{5}^{4}+z_{6}^{4}+z_{7}^{2}=0\right\}
$$


All representations of the rigid vacua and their mirrors that appear in the list of all LandauGinzburg vacua can in fact be mapped into each other via fractional transformations. The precise relations are collected in Table 1.

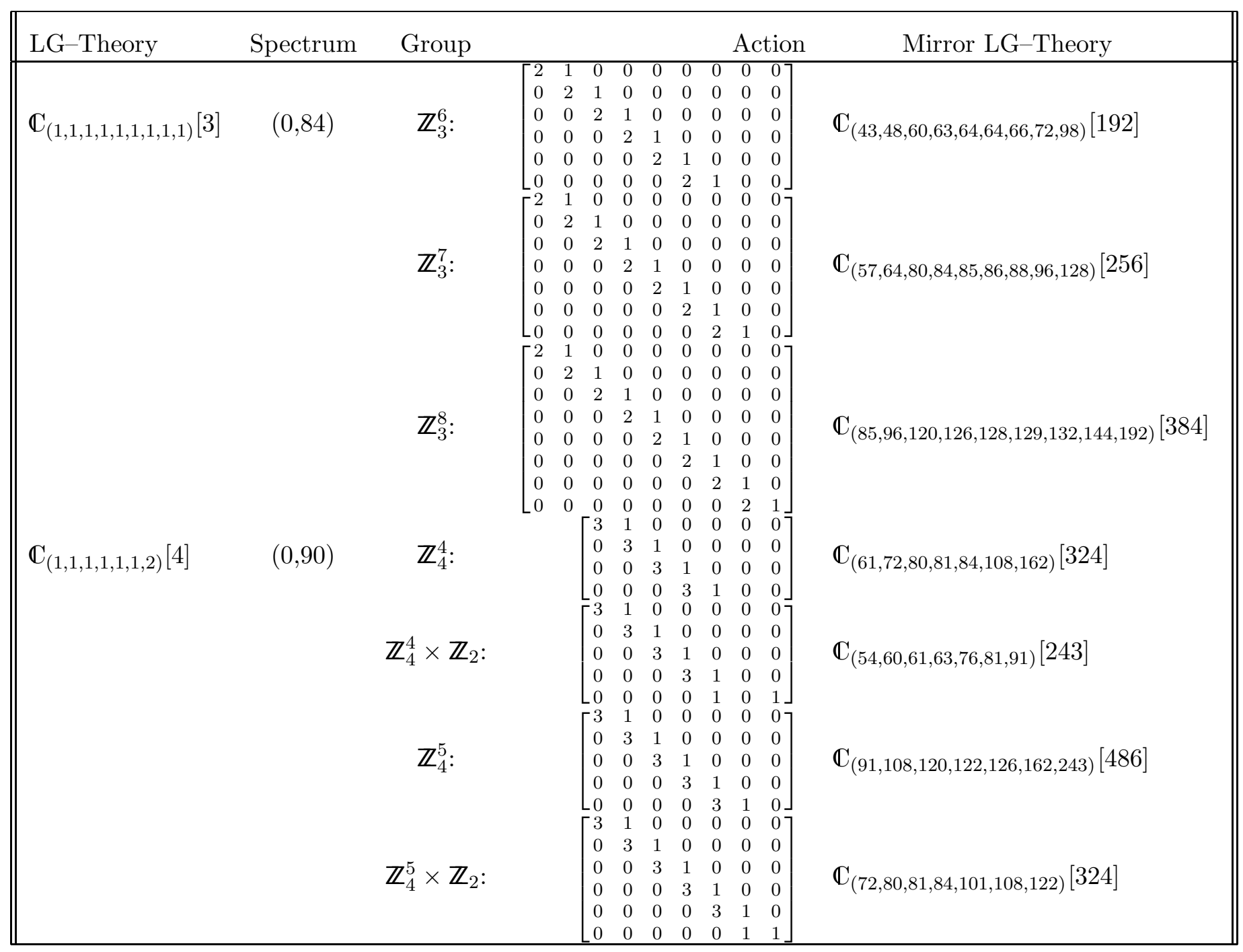

Table 1: Rigid Landau-Ginzburg Theories 用

Thus we have matched all the rigid vacua that appear in the construction of all LandauGinzburg theories [12] [13]. Aside from the rigid vacua that arise in the Landau-Ginzburg formulation of string vacua there exist of course also rigid vacua that are obtained by orbifolding

\footnotetext{
${ }^{4}$ This table contains the distinct theories. The other theories in the list of all Landau-Ginzburg vacua, listed here are isomorphic to the ones in Table 1.
} 
Landau-Ginzburg theories with respect to discrete groups. More importantly however there exist further rigid Calabi-Yau manifolds whose mirrors cannot be described in the Landau-Ginzburg framework. Examples are furnished by the resolution of particular nontransverse quintics constructed by Schoen [51] and van Straten [52].

Fractional transformations therefore suggest a detailed analysis of the special Fano varieties that are associated to the Landau-Ginzburg orbifolds in the table. More precisely it would be of interest to understand in detail the following pairings:

\begin{tabular}{||ll||}
\hline \hline Special Fano Varieties & Mirror Special Fano Varieties \\
\hline \hline $\mathbb{P}_{8}[3]$ & $\mathbb{P}_{(43,48,60,63,64,64,66,72,98)}[192]$ \\
& $\mathbb{P}_{(57,64,80,84,85,86,88,96,128)}[256]$ \\
& $\mathbb{P}_{(85,96,120,126,128,129,132,144,192)}[384]$ \\
\hline $\mathbb{P}_{(1,1,1,1,1,1,2)}[4]$ & $\mathbb{P}_{(61,72,80,81,84,108,162)[324]}[343]$ \\
& $\mathbb{P}_{(54,60,61,63,76,81,91)}[243]$ \\
& $\mathbb{P}_{(91,108,120,122,126,162,243)}[486]$ \\
& $\mathbb{P}_{(72,80,81,84,101,108,122)}[324]$ \\
\hline \hline
\end{tabular}

Table 2: Rigid mirror pairings in the framework of special Fano folds.

\section{Toric Considerations}

The simplest of all Calabi-Yau spaces surely is the set

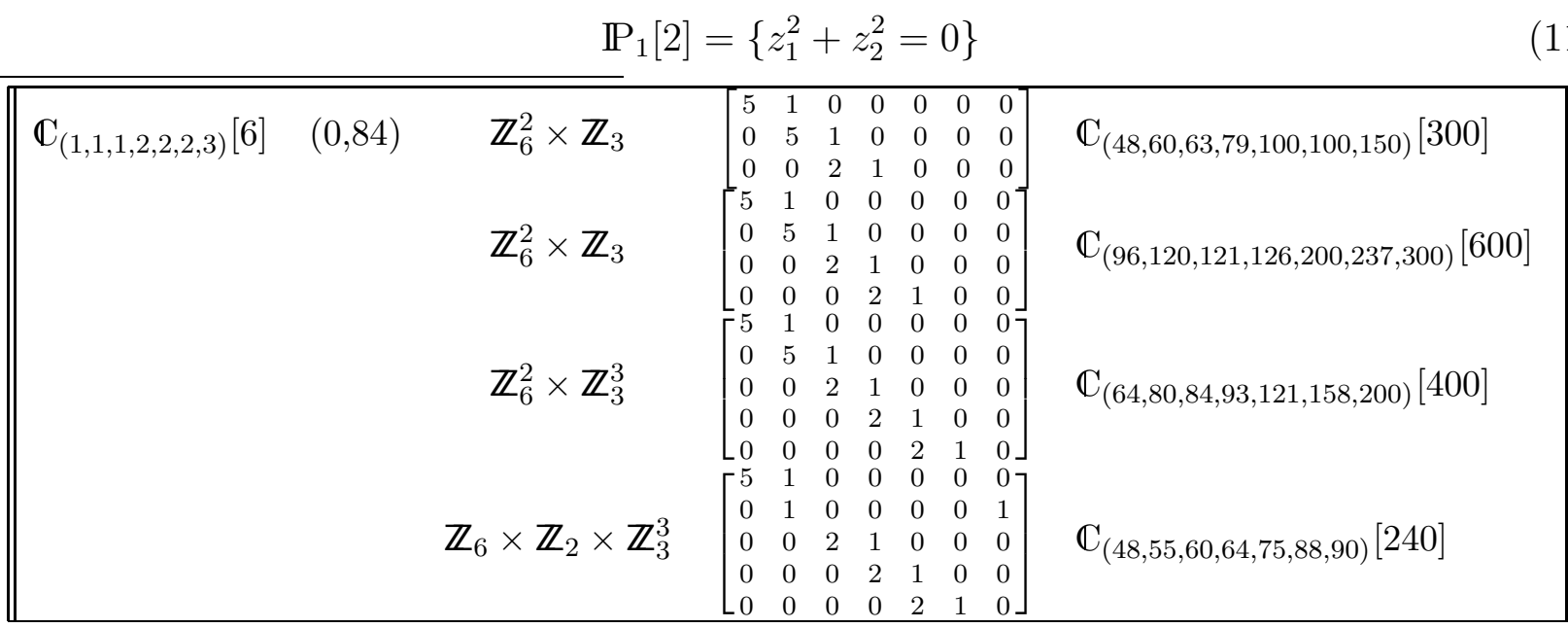


which happens to consist of two points exactly. The higher dimensional special Fano manifold of this space is $\mathbb{P}_{3}[2]$ whose toric analysis is what follows. This clearly is just a toy example, but it is a toy example of some consequence.

Writing

$$
\begin{aligned}
p & =x_{1}^{2}+\cdots+x_{4}^{2}+x_{1} x_{2}+\cdots x_{3} x_{4} \\
& =z_{3} z_{4}\left(\frac{z_{1}^{2}}{z_{3} z_{4}}+\frac{z_{2}^{2}}{z_{3}}+\frac{z_{3}}{z_{4}}+\frac{z_{4}}{z_{3}}+\frac{z_{1} z_{2}}{z_{3} z_{4}}+\frac{z_{1}}{z_{4}}+\frac{z_{1}}{z_{3}}+\frac{z_{2}}{z_{3}}+1\right)
\end{aligned}
$$

leads (in $U_{4}=\left\{x_{4}=1\right\}$ ) to the integral points

$$
(2,0,-1),(0,2,-1),(0,0,1),(0,0,-1),(1,1,-1),(1,0,0),(1,0,-1),(0,1,0),(0,1,-1),(0,0,0)
$$

which span a polyhedron whose vertices are

$$
\Delta=\{(2,0,-1),(0,2,-1),(0,0,1),(0,0,-1)\}
$$

This polyhedron has no integral point in its interior and hence one deduces from it that $h^{(2,0)}=0$ for the quadratic surface. This is good (and something we knew because the surface has positive first Chern class.) To obtain

$$
h^{(1,1)}(\bar{Z})=l^{*}(2 \Delta)-4 l^{*}(\Delta)-3-\sum_{\operatorname{codim} \Gamma=1}\left(l^{*}(\Gamma)-1\right)
$$

the first task is to count the number of integral points in the interior of the polyhedron $2 \Delta$ which is spanned by

$$
2 \Delta=\{(4,0,-2),(0,4,-2),(0,0,2),(0,0,-2)\}
$$

After some computing one finds that there is only one interior integral point $(1,1,-1)$ and hence

$$
l^{*}(2 \Delta)=1
$$

There are 4 two-dimensional faces with no interior integral points and therefore

$$
h^{(1,1)}(\bar{Z})=1-3-4(-1)=2
$$

which we also knew because $\mathbb{P}_{3}[2]=\mathbb{P}_{1} \times \mathbb{P}_{1}$.

The upshot of this toric analysis of the noncritical manifolds suggests that the special Fano manifolds defined in (2.4) lend themselves to a toric description in terms of polyhedra $\Delta$ which are characterized by the condition that the $Q$-fold has precisely one interior integral point

$$
l^{*}(Q \Delta)=1
$$


A much more detailed translation of our framework into the language of toric geometry has been provided by Batyrev and Borisov [41].

\section{Phases of Special Fano Manifolds}

It clearly is of interest to find a $\sigma$-model theoretic framework for our construction. In [53] an analysis of gauged $\mathrm{N}=2$ supersymmetric theories has been presented which also applies to models that do not correspond to Calabi-Yau spaces. This analysis pertains to models which may be closely related to special Fano manifolds. In the following attention is restricted to smooth manifolds; these contain, as we have seen, manifolds describing mirrors of rigid Calabi-Yau manifolds and therefore have been the most puzzling.

The starting point of the analysis in [53] is a $\mathrm{U}(1)$ gauge theory in $\mathrm{N}=2$ superspace, extending the standard Landau-Ginzburg action for the chiral N=2 superfields to

$$
L=L_{k i n, V}+L_{k i n, \Phi_{i}}+L_{W, \Phi_{i}}+L_{D, \theta} .
$$

Consider, in the notation of [53], the gauge invariant field strength

$$
\Sigma=\frac{1}{2 \sqrt{2}}\left\{\overline{\mathcal{D}}_{+}, \mathcal{D}_{-}\right\}
$$

the kinetic term of which is given by

$$
\mathcal{A}_{g}=-\frac{1}{4 e^{2}} \int d^{2} z d^{4} \theta \bar{\Sigma} \Sigma .
$$

There are two possible interactions, the $\theta$ angle and the Fayet-Illiopoulos D-term. These can be written as

$$
\mathcal{A}_{\mathrm{D}, \theta}=\frac{i t}{2 \sqrt{2}} \int d^{2} z d \theta^{+} d \bar{\theta}^{-} \Sigma+h . c .
$$

where

$$
t=i r+\frac{\theta}{2 \pi}
$$

where $r$ is the coefficient of the $\mathrm{D}$-term.

To this are added $N$ chiral superfields with $\mathrm{U}(1)$-charge $Q_{i}$. The kinetic energy of these fields is chosen to be

$$
\mathcal{A}_{m}=\int d^{2} z d^{4} \theta \sum_{i} \Phi_{i} \Phi_{i}
$$


and the superpotential is assumed to be of gauge invariant form

$$
\mathcal{A}_{W}=-\int d^{2} z d^{2} \theta W\left(\Phi_{i}\right)-\text { h.c. }
$$

which is supersymmetric because the $\Phi_{i}$ are chiral and $W$ is holomorphic.

The constant part of the lowest components of the superfields $\Phi_{i}$ will be thought of as parametrizing the $n$-dimensional complex space $\mathbb{C}_{n}$, assuming, as is done in [53], that the Kähler metric in the kinetic term of the $\Phi_{i}$ should be flat.

The bosonic equations of motion for the auxiliary fields $D_{a}$ and $F_{i}$ become

$$
D=-e^{2}\left(\sum_{i} Q_{i}\left|\phi_{i}\right|^{2}-r\right)
$$

and

$$
F_{i}=\frac{\partial W}{\partial \phi_{i}}
$$

The bosonic potential that one obtains in terms of the matter fields $\phi_{i}$ and the auxiliary fields $D_{a}$ and $F_{i}$ is

$$
U\left(\phi_{i}, \sigma\right)=\frac{1}{2 e^{2}} D^{2}+\sum_{i}\left|\frac{\partial W}{\partial \phi_{i}}\right|^{2}+2|\sigma|^{2} \sum_{i} Q_{i}^{2}\left|\phi_{i}\right|^{2}
$$

Including the one-loop correction of the theory leads to a $\sigma$-dependent effective $r$

$$
r_{\text {eff }} \sim r+\frac{\sum_{i} Q_{i}}{2 \pi} \ln \left(\frac{|\sigma|}{\mu}\right) .
$$

Assume now that the superpotential takes the form

$$
W\left(\Phi_{i}\right)=\Phi_{0} \tilde{W}\left(\Phi_{1}, \ldots ., \Phi_{N}\right)
$$

where $\tilde{W}$ is a quasihomogeneous polynomial which is assumed to be transverse, i.e. the equations

$$
\frac{\partial \tilde{W}}{\partial \Phi_{i}}=0
$$

can only be solved at the origin.

With this potential the bosonic potential becomes

$$
U\left(\phi_{i}, \sigma\right)=\frac{1}{2 e^{2}} D^{2}+|\tilde{W}|^{2}+\left|\phi_{0}\right|^{2} \sum_{i}\left|\frac{\partial \tilde{W}}{\partial \phi_{i}}\right|^{2}+2|\sigma|^{2}\left(\sum_{i} k_{i}^{2}\left|\phi_{i}\right|^{2}+k_{0}^{2}\left|\phi_{0}\right|^{2}\right)
$$


with

$$
D=-e^{2}\left(\sum_{i} k_{i}\left|\phi_{i}\right|^{2}-k_{0} \phi_{0} \bar{\phi}_{0}-r_{\mathrm{eff}}\right) .
$$

Note that all terms in (12.14) are $\geq 0$. Thus in order to minimize the potential $U$ one has to minimize $D^{2}$ which means different things, depending on what the variable $r$ does. Assume now, that all chiral superfields $\phi_{i}$ have charge unity under the $\mathrm{U}(1)$ gauge group.

The $r_{\text {eff }}=r>0$ phase: In this case not all $\phi_{i}$ can be zero. But since the polynomial $p$ is transverse everywhere except at the origin this means, because $\partial \tilde{W}$ is nonzero for some $i$, that $\phi_{0}$ must be zero. Thus $D=0$ leads to

$$
\sum_{i=1}^{N} \bar{\phi}_{i} \phi_{i}=r
$$

This simply defines a sphere $S^{2 N-1} \subset \mathbb{C}_{N}$. Recalling that one has to mod out the U(1) gauge group and also that the sphere $S^{2 N-1}$ can be Hopf-fibered $S^{1} \longrightarrow S^{2 N-1} \longrightarrow \mathbb{P}_{N-1}$ leads to the condition that the constant bosonic components of $\Phi_{i}$ parametrize a projective space $\mathbb{P}_{N-1}=S^{2 N-1} / U(1)$. Furthermore the vanishing of $\tilde{W}$ leads to a geometry for the space of ground states described by a hypersurface embedded in $\mathbb{P}_{N-1}$.

The $r_{\text {eff }}=r<<0$ phase: In this case the vanishing of $D$ leads to $\phi_{0} \neq 0$ and hence the term $\left|\phi_{0}\right|^{2} \sum_{i}\left|\partial_{i} \tilde{W}\right|^{2}$ enforces that $\phi_{i}=0$ since this is the only place where the partials are allowed to vanish, because of transversality. This fixes the modulus of $\phi_{0}$ to be

$$
\left|\phi_{0}\right|=\sqrt{-r} N
$$

Because of the gauge invariance the classical vacuum is in fact unique, modulo these gauge transformations. Expanding around this vacuum leads to massless $\phi_{i}$ (for $N \geq 3$ ). To find the potential for these massless fields one has to integrate out the massive field $\phi_{0}$. Integrating out $\phi_{0}$ means setting $\phi_{0}$ to its expectation value. Thus the effective superpotential of the low energy theory is

$$
\tilde{W}=\sqrt{-r} W\left(\phi_{i}\right)
$$

The factor $\sqrt{-r}$ is inessential since it can be absorbed by rescaling the $\phi_{i}$. Since the origin is a multicritical point, this describes a Landau-Ginzburg theory. More precisely it describes a Landau-Ginzburg orbifold.

Applying this analysis to the two smooth higher dimensional manifold, $\mathbb{P}_{8}[3]$, with Hodge diamond 


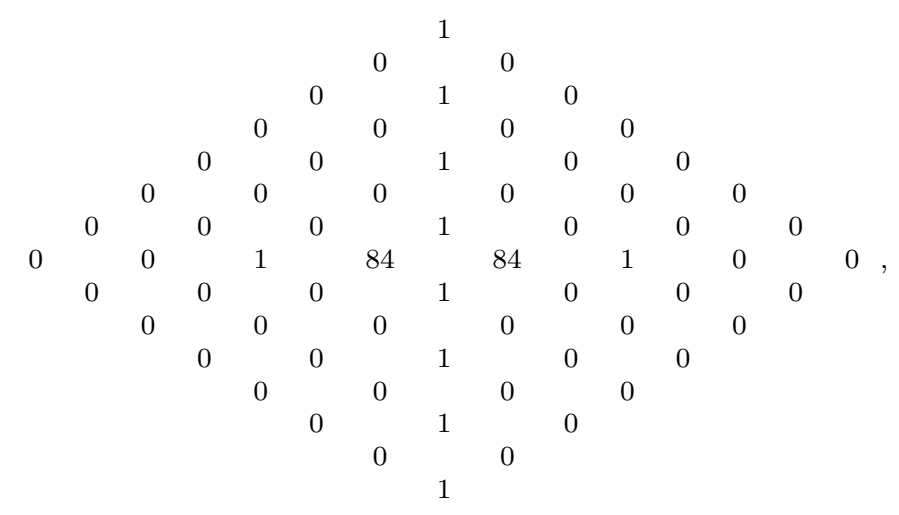

and $\mathbb{P}_{(1,1,1,1,1,1,2)}[4]$, with Hodge diamond

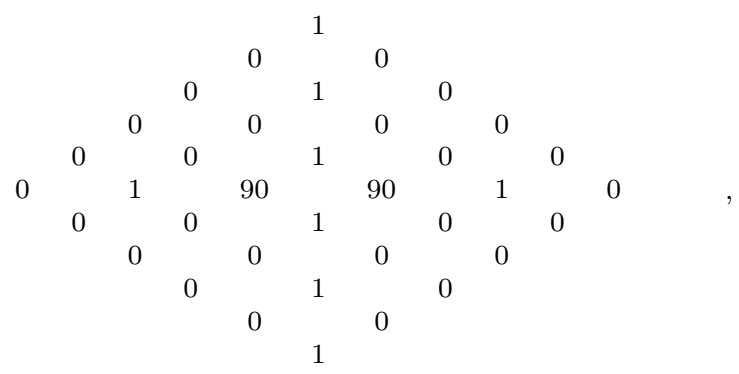

leads to the conclusion that there are two, respectively one, additional mode(s) in these theories that would not be present if we were to consider the relevant string spectrum. These numbers fit perfectly when considering the Hodge diamond decomposition of the cohomology of the higher dimensional special Fano manifold into a critical string part and a remaining part, as described in the previous Sections.

The above counting generalizes: for smooth special Fano manifolds of dimension $D_{\text {crit }}+2(Q-1)$ the vertical cohomology contains (for $D_{\text {crit }}=3$ )

$$
H^{(1,1)}, \ldots, H^{(Q, Q)}
$$

leaving $(Q-1)$ forms unaccounted for. Thus the counting of states described in 53 accounts precisely for the additional cohomology groups found in the special class of Fano folds (2.4) which suggests that it is the proper $\sigma$-model theoretic framework for these manifolds. 


\section{Special Complete Intersection Fano Manifolds}

The discussion in Section 3 of special Fano-folds which lead to critical manifolds of codimension three and larger indicates that the class of (2.4) admits a generalization to noncritical complete intersections.

More precisely, the analysis there suggests to consider the class of manifolds

$$
\begin{aligned}
& \mathbb{P}_{\left(k_{1}^{1}, \ldots, k_{n_{1}+1}^{1}\right)} \\
& \mathbb{P}_{\left(k_{1}^{2}, \ldots, k_{n_{2}+1}^{2}\right)} \\
& \quad \vdots \\
& \mathbb{P}_{\left(k_{1}^{F}, \ldots, k_{n_{F}+1}^{F}\right)} \\
& \mathbb{P}_{\left(k_{1}^{F+1}, \ldots, k_{n_{F+1}}^{F+1}\right)} \\
& \quad \\
& \mathbb{P}_{\left(k_{1}^{F+L}, \ldots, k_{n_{L}+1}^{F+L}\right)}
\end{aligned} \quad\left[\begin{array}{ccccccc}
d_{1}^{1} & d_{2}^{1} & \ldots & d_{f}^{1} & 0 & \ldots & 0 \\
d_{1}^{2} & d_{2}^{2} & \ldots & d_{f}^{2} & 0 & \ldots & 0 \\
\vdots & \vdots & \ddots & \vdots & 0 & \ldots & 0 \\
d_{1}^{F} & d_{2}^{F} & \ldots & d_{f}^{F} & 0 & \ldots & 0 \\
0 & \ldots & 0 & d_{f}^{F+1} & d_{f+1}^{F+1} & \ldots & d_{f+K}^{F+1} \\
\vdots & \ddots & \vdots & \vdots & \vdots & \ddots & \vdots \\
0 & \ldots & 0 & d_{f}^{F+L} & d_{f+1}^{F+L} & \ldots & d_{f+K}^{F+L}
\end{array}\right]
$$

such that

$$
c_{1}(M)=(Q-1-f) \sum_{i=f+1}^{f+K} c_{1}\left(\mathcal{N}_{i}\right)
$$

for some integer $Q \in \mathbb{N}$.

As an example consider the 7 -fold

$$
\mathbb{P}_{(5,6,4,5,6,4,4,4,6)}[16] \ni\left\{\sum_{i=1}^{2}\left(x_{i}^{2} y_{i}+y_{i}^{2} z_{i}+z_{i}^{4}\right)+y_{3}^{2} z_{3}+z_{3}^{4}=0\right\},
$$

the $\mathbb{Z}_{2}$-fibering of which leads to the weighted complete intersection

$$
\mathbb{P}_{1} \mathbb{P}_{(3,3,2,2,2,3,4)}\left[\begin{array}{ll}
2 & 0 \\
3 & 8
\end{array}\right]
$$

This variety is not a Calabi-Yau space since its first Chern class is nonvanishing

$$
c_{1}=8 h_{2},
$$

where $h_{2}$ is the pullback of the Kähler class of the weighted ambient factor. Furthermore, because $c_{1}(M)=c_{1}\left(N_{2}\right)$ it follows that $Q=3$ and the Calabi-Yau manifold is expected to be of codimension three.

Indeed, the $\mathbb{Z}_{3}$ fibering of this space leads to the space

$$
\begin{aligned}
& \mathbb{P}_{1} \\
& \mathbb{P}_{2} \\
& \mathbb{P}_{(1,1,1,2)}
\end{aligned} \quad\left[\begin{array}{lll}
2 & 0 & 0 \\
1 & 2 & 0 \\
0 & 1 & 4
\end{array}\right] \ni\left\{\begin{array}{l}
p_{1}=\sum_{i=1}^{2} x_{i}^{2} y_{i}=0 \\
p_{2}=\sum_{i=1}^{3} y_{i} z_{i}=0 \\
p_{3}=\sum_{j=1}^{3} z_{i}^{4}+z_{4}^{2}=0
\end{array}\right\}
$$


which does define a Calabi-Yau 3-fold of codimension 3.

A further simple example in this class is the noncritical manifold

$$
\begin{aligned}
& \mathbb{P}_{1} \\
& \mathbb{P}_{(1,1,1,2,2,2)}
\end{aligned}\left[\begin{array}{ll}
2 & 0 \\
1 & 4
\end{array}\right]
$$

with first Chern class

$$
c_{1}=4 h_{2} .
$$

Applying the paradigm leads to the critical complete intersection

$$
\begin{aligned}
& \mathbb{P}_{1} \\
& \mathbb{P}_{2} \\
& \mathbb{P}_{2}
\end{aligned}\left[\begin{array}{lll}
2 & 0 & 0 \\
1 & 2 & 0 \\
0 & 1 & 2
\end{array}\right]
$$

which describes a K3 surface.

\section{Conclusion}

It has been clear for some time now that, because of the existence of rigid (2,2)-string vacua mirror symmetry cannot be understood in the framework of Calabi-Yau manifolds. Instead there must exist, beyond the realm of such spaces, a new type of variety which does contain information about critical vacua, such as the spectrum of the massless fields, for both, Calabi-Yau type ground states as well as for vacua which describe mirrors of rigid Calabi-Yau manifolds.

It has been shown in [18] and the present paper that the special class of Fano manifolds of type (2.4) generalizes the framework of Calabi-Yau vacua in the desired way: For particular types of such Fano manifolds Calabi-Yau spaces of critical dimension are embedded algebraically in a fibered submanifold. For string vacua which cannot be described by Kähler manifolds and which are mirror candidates of rigid Calabi-Yau manifolds the higher dimensional manifolds still lead to the spectrum of the critical vacuum and a rationale emerges that explains why a Calabi-Yau representation is not possible in such theories. Thus these manifolds of dimension $c / 3+2(Q-1)$ define an appropriate framework in which to discuss mirror symmetry.

One intriguing consequence that follows from our results suggests the generalization of a conjecture regarding the relation between $(2,2)$ superconformal field theories of central charge $c=3 D, D \in \mathbb{N}$, with $\mathrm{N}=1$ spacetime supersymmetry on the one hand and Kähler manifolds of 
complex dimension $D$ with vanishing first Chern class on the other. It was suggested by Gepner [6] that this relation is $1-1$. Our analysis indicates that instead superconformal theories of such a type are in correspondence with Kähler manifolds of dimension $c / 3+2(Q-1)$ with a first Chern class quantized in multiples of the degree of the defining polynomial.

The results described here indicate that Fano manifolds of the special type considered in this paper are not just auxiliary devices but may be as physical as Calabi-Yau manifolds of critical dimension.

\section{Acknowledgement}

Part of this work was done at the Institute for Theoretical Physics, Santa Barbara, supported in part by NSF grant PHY-89-04035, the Theory Group at the University of Texas, and the Aspen Institute for Physics. I'm grateful to these institutions for support and hospitality and to V.V. Batyrev, P. Berglund, P. Candelas, E. Derrick, M. Lynker, A. Strominger and E. Witten for discussions.

\section{References}

[1] P. Candelas, M.Lynker and R.Schimmrigk, Nucl.Phys. B341(1990)383

[2] M.Lynker and R.Schimmrigk, Phys.Lett. B249(1990)237

[3] B.R.Greene and R.Plesser, Nucl.Phys. B338(1990)15

[4] P.Candelas, G.Horowitz, A.Strominger and E.Witten, Nucl.Phys.B258(1985)46

[5] D.Gepner, Nucl.Phys. B296(1988)757

[6] D.Gepner, Phys.Lett. B199(1987)380

[7] D.Gepner, String Theory on Calabi-Yau Manifolds: The Three Generation Case, Princeton University preprint, December 1987, hep-th/9301089

[8] E.Martinec, Phys.Lett. 217(1989)431 
[9] C.Vafa and N.Warner, Phys.Lett. 218(1989)51

[10] M.Lynker and R.Schimmrigk, in preparation

[11] M.Kreuzer, R.Schimmrigk and H.Skarke, Nucl.Phys. B372(1992)61

[12] A.Klemm and R.Schimmrigk, Nucl.Phys. B411(1994)559

[13] M.Kreuzer and H.Skarke, Nucl.Phys. B388(1992)113

[14] M.Kreuzer and H.Skarke, Phys.Lett. B318(1993)305; Nucl.Phys. B405(1993)305

[15] P.Berglund and T.Hübsch, Nucl.Phys. B393(1993)377

[16] M.Kreuzer, Phys.Lett. B314(1993)31 ; The Mirror Map for Invertible LG Models, CERNTH.7165/94 preprint

[17] P.Berglund and M.Henningson, Landau-Ginzburg Orbifolds, Mirror Symmetry and the Elliptic Genus, IASSNS-HEP-93/92 preprint

[18] R.Schimmrigk, Phys.Rev.Lett. 70(1993)3688

[19] P.Candelas, X.de la Ossa, P.Green and L.Parkes, Phys.Lett B258(1991)118; Nucl.Phys. B359(1991)21

[20] R.D'Auria and S.Ferrara, String Quantum Symmetries from Picard-Fuchs Equations and their Monodromy, CERN-TH-6777/93 and POLFIS-TH.24/93 preprints

[21] A.Ceresole, R.D'Auria and T.Regge, Duality Group for Calabi-Yau 2-Moduli Space, POLFISTH. 05/93 and DFTT 34/93 preprints

[22] M.Cadoni, String Quantum Symmetries and the SL(2,ZZ) Group, INFN-CA-93-12 preprint

[23] P.Aspinwall, B.R.Greene and D.R.Morrison, Phys.Lett. B303(1993)249; Calabi-Yau Moduli Space, Mirror Manifolds and Spacetime Topology Change in String Theory, IASSNSHEP-93/08 and CLNS-93/1236 preprints Measuring Small Distances in N=2 Sigma Models, IASSNS-HEP-93/49

[24] G.Ellingsrud and S.A.Stromme, in Essays on Mirror Symmetry, ed. S.-T.Yau, Int.Press.Co., Hong Kong 1992

[25] D.R.Morrison, in Essays on Mirror Symmetry, ed. S.-T.Yau, Int.Press.Co., Hong Kong 1992 
[26] A.Font, Nucl.Phys. B391(1993)358

[27] A.Klemm and S.Theisen, B389(1993)153

[28] A.Klemm and S.Theisen, Mirror Maps and Instanton Sums for Complete Intersections in Weighted Projective Space, LMU-TPU-93-08 preprint

[29] A.Libgober and J.Teitelbaum, Duke Math.J.Int.Math.Res.Notices 1(1993)29

[30] V.V.Batyrev and D. van Straten, Generalized Hypergeometric Functions and Rational Curves on Calabi-Yau Intersections in Toric Varieties, Essen University and Kaiserslautern University peprints

[31] P.Candelas, X. de la Ossa, A.Font, S.Katz and D.R.Morrison, Mirror Symmetry for Two Parameter Models I, UTTG-15-93, NEIP-93-005 and OSU-M-1 preprints

[32] S.Hosono, A.Klemm, S.Theisen and S.-T.Yau, Mirror Symmetry, Mirror Map and Applications to Calabi-Yau Hypersurfaces, Harvard preprint HUTMP-93/0801

[33] P.Candelas, A.Font, S.Katz and D.R.Morrison, Mirror Symmetry for Two Parameter Models II, UTTG-26-93, IASSNS-HEP-94/12 and OSU-M-94-1 preprints

[34] P.Berglund, E.Derrick, T.Hübsch and D.Jancic, On Periods for String Compactifications, UTTG-27-93, IASSNS-HEP-93/80 and HUPAPP-93/6 preprints

[35] M.Bershadsky, S.Cecotti, H.Ooguri and C.Vafa, Holomorphic Anomalies in Topological Field Theories, with an Appendix by S.Katz, Intersection Theory over Moduli Spaces of Degenerate Instantons, HUTP-93/A008

[36] M.Bershadsky, S.Cecotti, H.Ooguri and C.Vafa, Kodaira-Spencer Theory of Gravity and Exact Results for Quantum String Amplitudes, HUTP-93/A025

[37] B.R.Greene, D.R.Morrison and M.R.Plesser, Mirror Manifolds in Higher Dimension, IAS preprint, IASSNS-HEP-94/2

[38] S.Katz, Rational curves on Calabi-Yau manifolds: verifying predictions of Mirror Symmetry, Oklahoma State University preprint OSU-M-92-3

[39] B.Zumino, Phys.Lett. B87(1979)203

[40] P.Candelas, E.Derrick and L.Parkes, Nucl.Phys. B407(1993)115 
[41] V.V.Batyrev and L.A.Borisov, Dual Cones and Mirror Symmetry for Generalized Calabi-Yau Manifolds, Essen University preprint

[42] V.V.Batyrev, Dual Polyhedra and Mirror Symmetry for Calabi-Yau Hypersurfaces in Toric Varieties, University of Essen preprint, to appear in J.Algebraic Geometry

[43] P.Candelas, A.Dale, C.A.Lütken and R.Schimmrigk, Nucl.Phys. B298(1988)493

[44] C.Vafa, in Essays on Mirror Symmetry, ed. S.-T.Yau, Int.Press.Co., Hong Kong 1992

[45] B.R.Greene, C.Vafa and N.Warner, Nucl.Phys. B324(1989)371

[46] R.Schimmrigk, Phys.Lett. B193(1987)175; Nucl.Phys. B342(1990)231

[47] R.Schimmrigk, Phys.Lett. B229(1989)227

[48] E.Martinec, in Physics and Mathematics of Strings, Knizhnik Memorial Volume, eds. L.Brink et.al.

[49] C.Vafa, Mod.Phys.Lett. A4(1989)1169

[50] P.Green and T.Hübsch, Comm.Math.Phys. 113(1987)505

[51] C.Schoen, Reine und Angewandte Math. 346(1985)85

[52] D. van Straten, Topology, 32(1993)857

[53] E.Witten, Nucl.Phys. B403(1993)159 